\title{
ASTRO - A LOW-COST, LOW-POWER CLUSTER FOR CPU-GPU HYBRID COMPUTING USING THE JETSON TK1
}

\author{
A Thesis \\ presented to \\ the Faculty of California Polytechnic State University, \\ San Luis Obispo
}

\author{
In Partial Fulfillment \\ of the Requirements for the Degree \\ Master of Science in Computer Science
}

by

Sean Sheen

June 2016 
(c) 2016

Sean Sheen

ALL RIGHTS RESERVED 


\section{COMMITTEE MEMBERSHIP}

TITLE:

AUTHOR:

DATE SUBMITTED: June 2016

COMMITTEE CHAIR: Chris Lupo, Ph.D.

Associate Professor of Computer Science

COMMITTEE MEMBER: John Bellardo, Ph.D.

Associate Professor of Computer Science

COMMITTEE MEMBER: John Oliver, Ph.D.

Associate Professor of Computer Engineering 


\begin{abstract}
Astro - A Low-Cost, Low-Power Cluster for CPU-GPU Hybrid Computing using the Jetson TK1

Sean Sheen
\end{abstract}

With the rising costs of large scale distributed systems many researchers have began looking at utilizing low power architectures for clusters. In this paper, we describe our "Astro" cluster, which consists of 46 NVIDIA Jetson TK1 nodes each equipped with an ARM Cortex A15 CPU, 192 core Kepler GPU, 2 GB of RAM, and 16 GB of flash storage. The cluster has a number of advantages when compared to conventional clusters including lower power usage, ambient cooling, shared memory between the CPU and GPU, and affordability. The cluster is built using commodity hardware and can be setup for relatively low costs while providing up to 190 single precision GFLOPS of computing power per node due to its combined GPU/CPU architecture.

The cluster currently uses one 48-port gigabit Ethernet switch and runs Linux for Tegra, a modified version of Ubuntu provided by NVIDIA as its operating system. Common filesystems such as PVFS, Ceph, and NFS are supported by the cluster and benchmarks such as HPL, LAPACK, and LAMMPS are used to evaluate the system. At peak performance, the cluster is able to produce 328 GFLOPS of double precision and a peak of $810 \mathrm{~W}$ using the LINPACK benchmark placing the cluster at 324th place on the Green500. Single precision benchmarks result in a peak performance of 6800 GFLOPs.

The Astro cluster aims to be a proof-of-concept for future low power clusters that utilize a similar architecture. The cluster is installed with many of the same applications used by top supercomputers and is validated using the several standard supercomputing benchmarks. We show that with the rise of low-power CPUs and GPUs, and the need for lower server costs, this cluster provides insight into how 
ARM and CPU-GPU hybrid chips will perform in high-performance computing. 


\section{ACKNOWLEDGMENTS}

Thanks to:

- Chris Lupo for his guidance in designing, building, and validating the Astro Cluster.

- Gavin Baker for his help building the Astro Cluster 


\section{TABLE OF CONTENTS}

Page

LIST OF TABLES . . . . . . . . . . . . . . . . . . .

LIST OF FIGURES . . . . . . . . . . . . . . . . . . xi CHAPTER

1 Introduction . . . . . . . . . . . . . . . . . 1

2 Background and Related Works . . . . . . . . . . . . . . . 3

2.1 Power Performance Ratio . . . . . . . . . . . . . . . 3

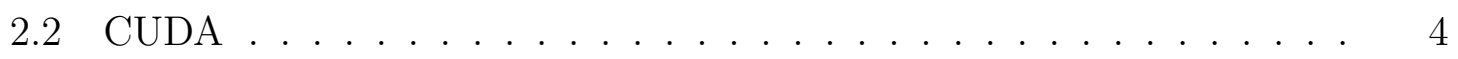

2.3 BLAS and cuBLAS Libraries. . . . . . . . . . . . 5

2.4 NVIDIA Jetson TK1 . . . . . . . . . . . . . . . . . 5

2.5 MPI Libraries . . . . . . . . . . . . . . . . . . . . . 6

2.6 OpenMP ............................. 7

2.7 Distributed File systems . . . . . . . . . . . . . . . . . 7

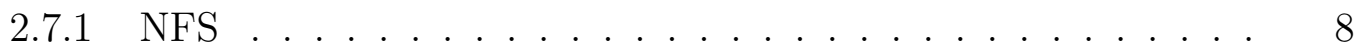

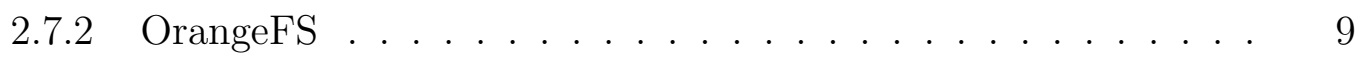

2.7.3 Lustre / Ceph . . . . . . . . . . . . . . . 10

2.8 Benchmarks . . . . . . . . . . . . . . . . . 11

2.8.1 High Performance Linpack . . . . . . . . . . . . . 11

2.8.2 LAMMPS Molecular Dynamics Simulator . . . . . . . . . 11

2.8.3 SPEC Power . . . . . . . . . . . . . . . . . 12

2.8.4 iPerf ............................... 12

2.9 Related Works . . . . . . . . . . . . . . . . . 13

3 Implementation . . . . . . . . . . . . . . . . . . 15

3.1 System Architecture and Design . . . . . . . . . . . . . . 15

3.2 Requirements . . . . . . . . . . . . . . . 15

3.2.1 Jetson TK1 . . . . . . . . . . . . . . . . . 15

3.2 .2 Low Power. . . . . . . . . . . . . . . 16

3.2 .3 Low Cost . . . . . . . . . . . . . . . 16

3.2.4 High Performance . . . . . . . . . . . . 16 
$3.2 .5 \quad$ Scalability . . . . . . . . . . . . . . . . 17

3.3 Hardware . . . . . . . . . . . . . . . . . . . . . . . . . . . . . 17

3.3 .1 Power Supply . . . . . . . . . . . . . . . . . . . . 17

$3.3 .2 \quad$ Server Rack . . . . . . . . . . . . . . . . . . . . . . . 18

3.3.3 Hardware Network Setup . . . . . . . . . . . . . . . . . . . 19

3.3.4 Hardware Summary . . . . . . . . . . . . . . . . . . . . . . 21

3.4 Software . . . . . . . . . . . . . . . . . . . . . 23

3.4 .1 Operating System . . . . . . . . . . . . . . . . . 23

3.4.2 Software Network Setup . . . . . . . . . . . . . . . . . . 23

3.4 .3 Cluster Management . . . . . . . . . . . . . . . . . . . . . 24

Managing Applications and Libraries . . . . . . . . . . . 24

Monitoring System Health and Stability . . . . . . . . . 25

3.4.4 Installed Packages and Libraries . . . . . . . . . . . . . . 28

3.4.5 File system Installation and Configuration . . . . . . . . 28

NFS . . . . . . . . . . . . . . . . . . . . . 28

OrangeFS v2.9 $\ldots \ldots \ldots \ldots \ldots$

Ceph (INFERNALIS) $\ldots \ldots \ldots \ldots$

3.5 Modifications to High Performance Linpack . . . . . . . . . 30

3.5.1 Removing Incompatible CUDA Functions _ . . . . . . . 30

3.5.2 Modifying Memory Usage . . . . . . . . . . . . . . . . 31

3.5.3 Configuring the HPL benchmark . . . . . . . . . . . 33

3.6 Modifying the LAMMPS Benchmark . . . . . . . . . . . 33

3.6.1 Configuring the LAMMPS Benchmark . . . . . . . . . . 34

3.7 Shortcomings of the System . . . . . . . . . . . . . . . . . . 34

3.7 .1 Limited File System Support _. . . . . . . . . . . . . . 34

3.7 .2 Performance Tools . . . . . . . . . . . . . . . . . . 35

3.7 .3 Network . . . . . . . . . . . . . . . . . . . . . . . 35

4 Results. . . . . . . . . . . . . . . . . . . 36

4.1 Experimental Setup . . . . . . . . . . . . . . . . 36

4.1 .1 Cluster $\operatorname{Setup~\ldots .~.~.~.~.~.~.~.~.~.~.~.~.~} 36$

4.1 .2 Measurement Tools . . . . . . . . . . . . . . . . . . . . 36

4.2 Power and Temperature $\ldots \ldots \ldots \ldots \ldots \ldots$ 
4.3 Network . . . . . . . . . . . . . . . . . . . . . . . . . 39

4.4 Memory Bandwidth . . . . . . . . . . . . . . . . . . . 39

4.5 Single-Node Performance . . . . . . . . . . . . . . . . . 41

4.6 Cluster Performance . . . . . . . . . . . . . . . . . . . . . 42

5 Conclusion . . . . . . . . . . . . . . . . . . . . 55

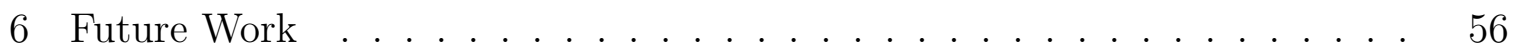

BIBLIOGRAPHY . . . . . . . . . . . . . . . . . 57 APPENDICES

A DHCP Configuration . . . . . . . . . . . . . 62

B Ganglia Configuration ................. 64

C Cluster Scripts ...................... 65 


\section{LIST OF TABLES}

Table

Page

3.1 Summary of Hardware components . . . . . . . . . . . . . 22

3.2 NVIDIA profiler summary for HPL with memcpy . . . . . . . . . 31

3.3 NVIDIA profiler summary for HPL with zero-copy . . . . . . . . . 32

4.1 Input Values for HPL benchmark . . . . . . . . . . . . . . 44

4.2 Input Values for LAMMPs benchmark . . . . . . . . . . . . . 48 


\section{LIST OF FIGURES}

Figure

3.1 Power Rack in the cluster . . . . . . . . . . . . . . . . 18

3.2 Power supply rails for each layer . . . . . . . . . . . . . . . . . . . . 19

3.3 Completed Server Rack (46 nodes) . . . . . . . . . . . . . 20

3.4 Switch setup for the Astro cluster . . . . . . . . . . . . . . . . 22

3.5 Ganglia web frontend main view . . . . . . . . . . . . 26

3.6 Ganglia web frontend alternate view . . . . . . . . . . . 27

3.7 Power Meter used to monitor power usage of the cluster . . . . . . 27

4.1 Power usage of the cluster as utilization increases . . . . . . . . 38

4.2 Network Bandwidth in the Astro cluster . . . . . . . . . . . . . . . 40

4.3 Performance of various LAPACK routines for CPU/GPU . . . . . 43

4.4 Example input file for HPL benchmark: only N, P, and Q are modified for different node sizes . . . . . . . . . . . . . . . . . 45

4.5 HPL benchmark scaling run with both CPU and GPU only implementations ..................... 46

4.6 HPL benchmark power usage for both CPU and GPU . . . . . . . 47

4.7 Example input file for Lammps benchmark . . . . . . . . . . . . . 49

4.8 LAMMPS Benchmark GPU vs CPU runtimes . . . . . . . . . . 50

4.9 LAMMPS GPU benchmark with Communication Time . . . . . . 51

4.10 Run time with no Communication Times . . . . . . . . . . . . . 52

4.11 Parallel Efficiency of the LAMMPS benchmark . . . . . . . . . 53 


\section{Chapter 1}

\section{INTRODUCTION}

First introduced in the 1960s, A supercomputer is a computer with high-level computational capacity and its performance is often measured in floating-point operations per second. These supercomputers often include hundreds to thousands of computers hooked together in a high speed optical network. These systems are often used in computational sciences where certain computational tasks require more compute power than a general purpose computer can provide. By using numerous computers hooked together, a large tasks can be broken down into smaller components and the results of these smaller components can be combined to solve the original task.

In recent years numerous supercomputing systems have begun using General Purpose Graphic Processing Units (GPU) or other co-processor technologies in highperformance computing (HPC) applications. These systems generally have the GPU or co-processor attached on a PCIe bus and require data to be transferred to and from the device limiting the efficiency of these accelerator cards. Many of the top systems also require a significant amount of power and are extremely expensive to build and maintain. NVIDIA's Jetson TK1 uses an integrated memory system that allows both the CPU and the GPU to share memory, which eliminates data transfer costs when performing GPU tasks. Combined with the low-power architecture provided by the Cortex-A15 ARM processor, this board provides an impressive theoretical computeto-power ratio and a relatively low cost per board. The Astro cluster, consisting of 46 Jetson TK1 boards, aims to provide a cost-effective system for running distributed GPU tasks.

Performance lists such as the Green500 [28] have brought attention to the unsustainable power costs required for HPC clusters. With current technologies, exascale 
machines will require more than 100 megawatts of power resulting in a cost of 100 million dollars each year [38]. Power efficiency is becoming a real problem while the push for exascale computing continues. Many researchers have started to focus on more efficient cluster designs and new architectures such as the one provided by the Jetson TK1. These more power efficient components can provide insight into how future supercomputing clusters should be designed.

In this paper we present insight gained from the design, implementation, and testing of the Astro Cluster. While the Jetson TK1 boards may not provide the same speeds as many top supercomputers, the goal of this cluster is to demonstrate the viability of ARM-based, CPU-GPU hybrid clusters as power-efficient HPC systems.

Astro has been tested with a variety of benchmarks and applications to evaluate the performance of the cluster including network, file systems, Memory I/O, and compute speeds. The 46 node cluster consumes a total of $810 \mathrm{~W}$ of power at full load, costs 5000 dollars, and provides 6800 GFLOPs of computing power. The Astro cluster performs better than many top supercomputers in terms of cost per Flop as well as Kilowatts per Flop but does significantly worse in I/O speeds and storage space. 
Chapter 2

\section{BACKGROUND AND RELATED WORKS}

Building a supercomputer often involves a variety of components that differ depending on the goal of the machine. Some of the considerations when building a cluster are discussed in this section.

\subsection{Power Performance Ratio}

For many top supercomputers, power to performance ratio is becoming more important due to operation costs of large clusters. The goal of many large corporations in recent years has been to minimize costs of running large data centers without affecting their performance. Some estimates have shown that in the US, servers use up around $1.2 \%$ of all power consumption [19] with $80 \%$ of the power being used while the machines are idle as shown in [23]. Many techniques have been developed to try and reduce idle power including techniques such as Vary On Vary off [16], where machines are turned off when not in use to reduce power consumption.

While these techniques can be effective in some scenarios, most data centers operate in a middle ground where nodes are generally utilizing around 20-30\% of their CPU at all times [23]. Many studies have also shown that even at low utilization, power usage is similar to when the device is at full load [16]. Many low power clusters have been proposed that use different CPU architectures that provide much lower idle energy consumption and scale better with work load resulting in more efficient power usage. 


\subsection{CUDA}

CUDA is a framework for performing general purpose computing on NVIDIA GPUs (graphical processing units). These devices are equipped with hundreds of cores and are able to create many threads to perform parallel tasks better than on the CPU. In a general work-flow data is transferred to the device, is operated on in parallel and a solution is returned to the host machine. Many of the top supercomputers use either a GPU or a co-processor to achieve faster speeds [12].

The downside of using CUDA programming is often the memory transfer costs from the CPU to the GPU. For smaller problem sizes or problems that require frequent memory transfers the speedup provided by the GPU may be offset by the time required to perform the constant memory transfers and synchronizations.

CUDA code is often split up into two major parts, setup and the kernel. Setup involves managing memory to be used by the GPU, transferring memory to the GPU, and setting up correct thread layouts. The CUDA thread model contains 4 major

parts: the thread, block, grid and warp. Threads are grouped into blocks, and blocks grouped into a grid with each thread having a unique local index relative to the block which also has a unique index relative to its location in the grid. These values can be adjusted to suit the problem with some limitations depending on the type of GPU. Threads in a block can synchronize and share data only with other threads in the same block, if threads need to communicate with other blocks global memory must be used which is significantly slower and can greatly reduce the speed of a program. A warp is a subset of the threads in a block and determine the maximum amount of threads that can run in parallel. Adjusting these parameters is often a large part of tuning a GPU program.

The kernel is the actual code that will be ran on the GPU. The kernel will use the 
unique ids of threads and blocks generated by the setup and map them to values to perform computation. Once the kernel is complete, the CPU can copy the solution produced by the GPU back to the CPU. Generally kernels are ran sequentially but certain GPUs allow multiple kernels to be ran concurrently. NVIDIA also provides a set of kernels for common math routines in their cuBLAS library [32] which is used extensively in HPC applications.

\subsection{BLAS and cuBLAS Libraries}

Basic Linear Algebra Subprograms (BLAS) is a set of low-level routines for performing common linear algebra operations. These libraries are often used in HPC applications and benchmarks. Numerous implementations of these routines are available such as openBLAS [6], MKL [29], and ATLAS [27]. These libraries conform to a common BLAS interface allowing a developer to write programs that use these functions without specifying a specific library.

The cuBLAS library provided by NVIDIA provides the same BLAS routines that are optimized for the GPU. The cuBLAS library also provides a set of helper functions that help streamline memory transfers and allocations when setting up the GPU. However, the cuBLAS library only provides a subset of the routines available in common BLAS libraries and cannot be used as a drop in replacement for other BLAS libraries.

\subsection{NVIDIA Jetson TK1}

The Jetson TK1 is NVIDIA's first embedded Linux development platform featuring

the Tegra K1 SOC. Some of the main features of the Jetson TK1 include the Kepler GK20a 192 core GPU, ARM quad-core Cortex-A15 CPU, 2GB of RAM, and 16GB of 
fast eMMC storage. The TK1 also supports a SATA connection, mini-PCIe connection, and is cooled using a fan. The main advantages of using the TK1 in a clustered environment is its low power usage and high theoretical performance. Typical power consumption falls between 1 to 5 Watts and performance peaks can reach as high as 326 GFLOPS. The board is also easy to install and setup as it comes pre-installed with both the Linux4Tegra operating system and a modified Linux kernel. CUDA can also be easily installed on the board and at the time of this writing CUDA 6.5 is supported.

The biggest advantage of the Jetson TK1 is its ability to support zero-copy mechanisms in CUDA. As mentioned previously most CUDA programs required memory to be transferred to and from the GPU which can incur high overhead costs. The TK1 avoids this cost by having the GPU and CPU share the same memory space, instead of transferring memory from the CPU to the GPU, only the pointer of the memory needs to be transferred and no additional space needs to be allocated. By having this zero-copy mechanism the TK1 can support a wider variety of GPU programming due to the elimination of one of the biggest bottlenecks in CUDA programming.

\subsection{MPI Libraries}

The Message Passing Interface (MPI) is a standardized message passing system often used in clusters for parallel applications. Many implementations of MPI exist such as MPICH [5] and Open MPI [7].

These libraries allow programs to transfer data and information between machines while running parallel tasks. MPI provides functions that are capable of handling synchronization, job distribution, and simple map-reduce functionality.

An MPI program must first be initialized and given the number of machines being used. These machines are assigned a unique identifier which can be used for both 
point-to-point communication and broadcast. MPI provides a clean interface for network communications between cluster nodes and simplifies the distributed programming model by abstracting away the complicated network communication required to synchronize and send data between nodes.

Many implementations of the MPI library are also CUDA-aware and can support running CUDA kernels on multiple nodes. However, MPI does not support checkpointing or data recovery and will require a complete restart if any node fails or is corrupted during a job. MPI also requires libraries and binaries needed for the specific job to exist in the same directory on all machines which is commonly achieved through the use of a distributed file system.

\subsection{OpenMP}

OpenMP is an application programming interface that aids in shared memory multiprocessing and supports a wide array of architectures and operating systems [33]. OpenMP is portable, scalable, and is often used in conjunction with Open MPI to create scalable parallel programs for large clusters. OpenMP allows easy modification of threads using global variables (OMP_NUM_THREADS) and can be turned off or on without recompilation of the program. Many distributed applications and cluster benchmarks use OpenMP and the Astro Cluster is able to take advantage of OpenMP due to the quad-core cortex A15 ARM CPU available on the TK1.

\subsection{Distributed File systems}

When operating a cluster, files need to be consistent and available on all machines. A distributed file system aims to be transparent to the user and handles locating files and transporting files between machines automatically. Many distributed file systems 
exist such as NFS, PVFS [35], and Lustre [20]. These all providing a varying level of features. The Lustre file system is currently unavailable for ARM 32 bit systems and a Ceph [43] a file system that provides similar features is used as a replacement.

A distributed file system allows a cluster to distribute compiled applications, libraries, or any data file that may be used by all nodes. Input files required for specific jobs can also be easily distributed between nodes in a cluster and allows a cluster to scale up and down without having to manually synchronize files. Using a distributed file system greatly simplifies file management and distribution in a cluster environment while also providing reliability and data recovery for files. Some of the file systems used in the Astro Cluster are described below.

\subsubsection{NFS}

The Network File System (NFS) operates using TCP or UDP Internet protocols, without needing any specialized equipment or layers to operate on. Due to the multitude of different configurations uesed to send data over the Internet, NFS requests go through External Data Representation (XDR) to ensure that under different computer architectures, the data follows a single understood uniform format. For example, byte endian-ness and integer sizes are standardized under XDR to allow a common channel of communication between systems with different internals.

NFS is not a implementation of a file system, but rather a remote access to another system's file system. The client remotely mounts a directory under its own file system. The underlying implementation of the mounted directory is entirely dependent on the host's choice of file system.

Since the client simply remotely accesses the host's file system, the host will typically be storing all the metadata and data. The files are accessible by many clients, but the data is not distributed out, making NFS not a scalable file system. 
As the data store grows larger, the file system will bottleneck at the read or write speeds of the host, due to many clients connecting to one host. In comparison, a distributed file system with only one file data server will perform equivalently with NFS.

The Astro Cluster supports NFS clients by default with minor kernel changes to support NFS servers. A single server is used in the Astro Cluster and one directory is shared to all clients.

\subsubsection{OrangeFS}

OrangeFS is an open-source stateless scalable distributed file system. It is based on the Parallel Virtual File System (PVFS)[36]. OrangeFS distributes the data by having a metadata server and multiple data stores which contain file data.

OrangeFS can designate servers to serve metadata, file data, or both. OrangeFS can run in both the kernel-space and user-space. Running in user-space may add latency into the system, but allows for easier installation. Running OrangeFS in kernel-space will remove abstraction layer function calls, making the file system faster, but requires a supported kernel.

OrangeFS's core unit is an object. Each directory or file contains at least 2 objects, one being the metadata and the other containing the actual data. Each object in OrangeFS is composed of a unique handle, a key/value pair of metadata, and a byte stream of data that belong in the object. The handle is unique among the entire file system, and the server location of the file can be determined from the unique handle. When a client requests a file, the query is sent to all metadata servers. The metadata servers reply with the location of where the file data is. The file data may live in stripes located on different servers. The client then fetches all the stripes at the same time and delivers them to the client. 
OrangeFS only modifies and manages metadata and the distribution of data. The actual storage of the data is left for the server's own local file system.

The Astro Cluster supports PVFS based file systems by default and no kernel changes are required to install both the server and the client.

\subsubsection{Lustre / Ceph}

The Lustre file system is a parallel distributed file system intended for high performance computing environments and used by almost all top500 supercomputers. Lustre is currently not supported by the Astro Cluster due to no ARM support. A file system with similar features, Ceph, is used as a replacement.

The Ceph file system is a distributed file system that provides performance, reliability, and scalability by separating data and metadata management. While running the Ceph file system individual nodes can be setup as metadata, data, or IO nodes that all perform different tasks. This separation of functionality prevents a single node from being a bottleneck when using the file system. The main draw of the Ceph file system is its ability to scale data and metadata sizes independently. By allowing nodes to handle data replication, failure detection, and recovery independently without communicating with a central node, the file system becomes incredibly scalable as communication in the file system is not centralized.

The Ceph file system is still in its early stages but supports POSIX semantics and can be utilized using a kernel module or file system in user space (FUSE). More details of its implementation can be found at [43]. 


\subsection{Benchmarks}

Many benchmarks are available for assessing performance of cluster systems. Some of the benchmarks used by Top500 supercomputers are described below [12].

\subsubsection{High Performance Linpack}

The LINPACK benchmark is an industry standard benchmark used to measure a cluster's floating-point computing power. High Performance Linpack (HPL) is a portable implementation of the original LINPACK benchmark and is currently the most commonly used benchmark by top supercomputers.

The LINPACK benchmark measures how fast a computer can solve a dense system of linear equations, a common task in science and engineering computations. The benchmark aims to provide an approximate into how fast a cluster will perform when solving real world problems. The benchmark is highly scalable and has been used to benchmark single machines and some of the largest supercomputers in the world [12].

The HPL version used to benchmark the Astro Cluster is a modified version of HPL-2.2 provided by NVIDIA that has been modified to support CUDA. Sections of the original benchmark are intercepted and processed on the GPU with minimal changes to the original source code.

\subsubsection{LAMMPS Molecular Dynamics Simulator}

The LAMMPS benchmark [4] differs from the LINPACK benchmark in that it does not perform complicated matrix calculations but instead aims to simulate molecular dynamics in single precision. Numerous types of simulations are provided but the main benchmark used to test the Astro cluster is the Lennard-Jones Potential simulation. The Lennard-Jones potential aims to approximate the interactions between a 
pair of neutral atoms or molecules.

The LAMMPS benchmark provides a good test for network communications and parallel efficiency of the cluster due to the large amount of network communication required for the benchmark. Each node receives a subset of molecules in the simulation and must communicate with their neighbors continuously to determine the molecule interactions and adjust accordingly.

\subsubsection{SPEC Power}

The SPEC Power benchmark [17] is an industry standard benchmark that evaluates the power and performance characteristics of a cluster. The benchmark runs a java application that runs tasks that utilize varying amounts of the CPU and calculates power usage at different utilization levels. Prior to running the benchmark a set of rules need to followed to make sure the tests are consistent including compiler options and device configuration. The benchmark is currently incompatible with the Jetson TK1.

While the SPEC benchmark is not used to benchmark the Astro cluster, similar guidelines and configurations are used to provide an accurate power consumption

measurement for the Astro Cluster. Many of the SPEC benchmarks do not have CUDA versions available and would not be able to fully test the power utilization of the TK1.

\subsection{4 iPerf}

iPerf [3] is an open source tool used to test network bandwidth and latency in clusters. Data is transferred between machines and timed to calculate the throughput of the system. 
iPerf uses TCP and a configurable window and payload size to determine the bandwidth and latency between two machines. One machine acts as the host while the other acts as a client and a payload is transferred between the two machines. Duration of the transfer along with packet characteristics are monitored and bandwidth, latency, network jitter, and packet loss statistics are reported. Multiple clients and UDP benchmark tests are also supported.

\subsection{Related Works}

As seen from recent research, low power cluster systems are becoming increasingly popular with projects such as FAWN [11] showing the viability of a low power cluster designed for specific tasks. Their approach showed that with proper setup and hardware, a low power cluster can achieve up to 2 times more energy efficiency when compared to other systems that performed the same job. Other approaches such as the Iridis-pi cluster [14] show that an ARM CPU is capable of being used in HPC environments.

Since its release, the Jetson TK1 has been used in many applications unrelated to HPC. The Jetson TK1 has been shown to be effective for computer vision and robotics, and can provide significantly faster image processing speeds due to the integrated GPU, while still being usable for long durations due to its low power usage. This is shown in a recent UAV computer-vision project [42]. Other projects have also shown the Jetson's usefulness in video processing and rendering due to the unified memory and the removal of data transfer costs.

The Jetson TK1 uses many of the same architectural features present in those low power clusters, and has been shown to provide significant computing power. The Jetson TK1 and other ARM CPU / GPU hybrid boards are useful for building low-power clusters due to the low energy requirements and high compute capabilities. 
Although these low-power clusters may not achieve the same application performance as Top500 clusters, the goal is to provide a power efficient platform for distributed applications. Current supercomputers are rarely concerned with power usage and instead aim to provide as much computing power as possible. 
Chapter 3

IMPLEMENTATION

\subsection{System Architecture and Design}

This section begins with a list of requirements and goals for the cluster, then provides a description of the architecture of the Astro cluster including both software and hardware components. We detail the costs of the system minus power measurement tools along with potential improvements. The section concludes with some discussion on sacrifices made to keep the cluster at a low cost.

\subsection{Requirements}

Prior to building the Astro cluster, a set of specifications were made for the project. These requirements were used as a guideline while designing the hardware and software components of the cluster. The special requirements for the cluster are listed below.

\subsubsection{Jetson TK1}

The first and most important requirement was the use of the Jetson TK1 as the nodes of the cluster. The Jetson TK1 was chosen due to its novel architecture that combines a low power ARM CPU and Kepler GPU all in one package while also supporting unified memory access. The board also supports a PCI-express connection and a SATA port which could be used to upgrade the cluster with more storage space or network capacity if needed. The Jetson TK1 was at the time, a very interesting development board with high theoretical performance and was well suited for testing 
ARM / GPU architectures in a clustered environment.

\subsubsection{Low Power}

The next requirement of the Astro cluster is to perform normal distributed and HPC tasks using minimal power with minimal impact on performance. Taking advantage

of the low power ARM CPU and the addition of a GPU, the Astro cluster should be able to achieve a high power to performance ratio and any techniques that can reduce power usage without hindering performance should be utilized. This involves removing any hardware or software components that are not used by the system.

\subsubsection{Low Cost}

While the cost of the cluster was largely controlled by the hardware used in the system, the goal was to reduce costs when possible either by reducing the amount of hardware required or using off-the-shelf commodity hardware that was cheaper and easier to modify. Lower costs for a cluster will ultimately be the result of using less power which was the primary goal of this cluster.

\subsubsection{High Performance}

The performance of the cluster should also be near its theoretical value. While modifications to the cluster were mainly aimed at reducing power and costs, changes should be made to increase the performance as well. This could include using faster network switches, better file systems, or taking advantage of hardware accelerators (such as the GPU) when possible. 


\subsubsection{Scalability}

Due to the relatively small size of the cluster, the ability to add more nodes and scale up the system was important in case more boards were available in the future. Without proper planning adding new boards may become difficult due to either network or power management. Designing a scalable cluster will be important for possible cluster expansion in the future.

\subsection{Hardware}

With those requirements in mind, the Astro cluster was composed of 46 Jetson TK1 development boards, 1 Gigabit network switch, a custom built server rack, 2 off-theshelf 500W bronze power supplies, and other miscellaneous items needed to construct the cluster. More expensive alternatives for the switch and power supply were available but exceeded the budget for the cluster.

\subsubsection{Power Supply}

Two off-the-shelf 500W bronze PC power supplies are used to power the entire cluster minus the switch and other monitoring equipment such as the power meter. The power supplies are heavily modified to support the TK1 and the original connections are removed and bundled together to create multiple rails that can support up to 100W per rail. Each of these rails are pulled down through the center of a rack layer containing boards and connected to the Jetson TK1 using a normal barrel connector. The rails were made using 24 gauge wire due to the large amount of current going through the wire while the connections to the individual nodes were 8 gauge. Figure 3.1 shows the power supply layer in the cluster. Three power supplies allow the cluster ti support up to 92 nodes. The rails going to each layer are shown in Figure 3.2, each 


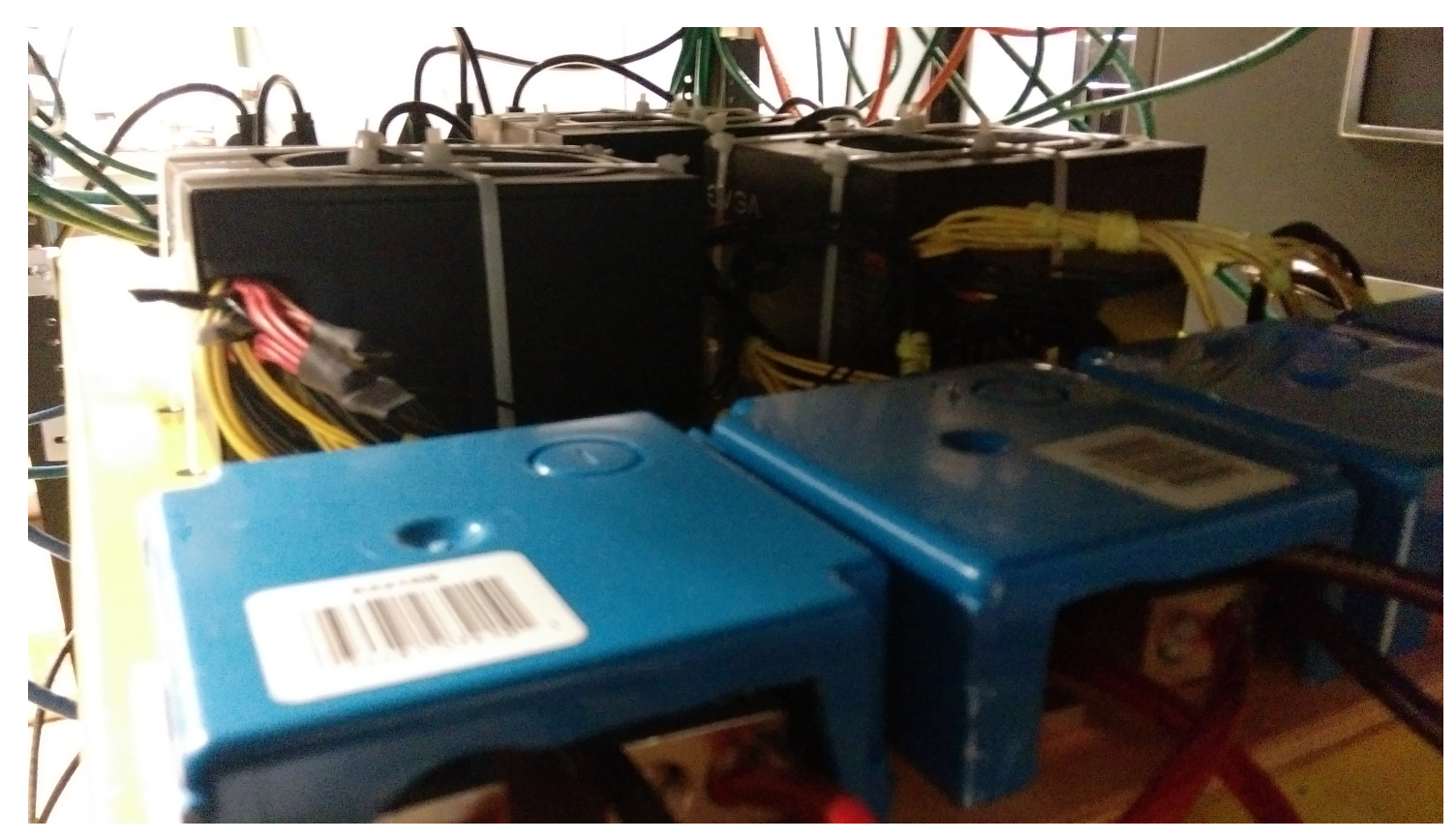

Figure 3.1: Power Rack in the cluster

rail currently powers 12 boards, but can support up to 16 .

When designing this setup we chose to use normal PC power supplies due to their ease of purchase and modification. PC power supplies are also safe when shorted and simply shut off which can prevent damage to the system when accidents occur. A bronze rating was chosen due to its lower cost and efficiency not being crucial to our system due to the constant high loads drawn by the cluster as apposed to silver or gold rated power supplies that are more efficient.

The cost of setting up the power supplies included 200 feet of 14 gauge wire, 25 feet of 8 gauge wire, and 46 barrel plugs.

\subsubsection{Server Rack}

The server rack that houses the Astro cluster is an $8 \mathrm{U}$ server rack with custom mounts for the Jetson TK1 boards. Each layer in the rack houses either a switch, up to 12 boards, or the 2 power supplies used in the system. 


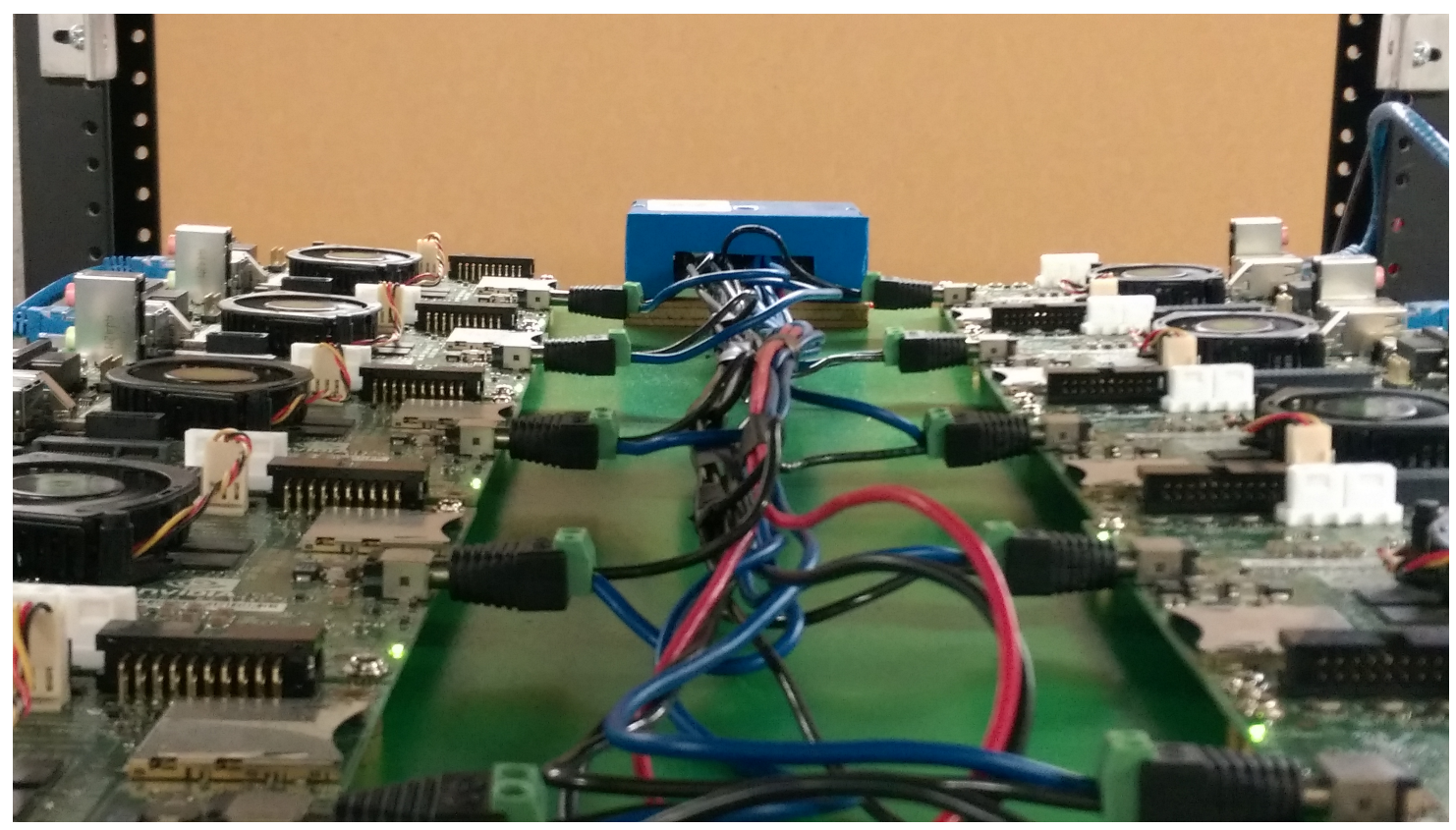

Figure 3.2: Power supply rails for each layer

The design of the rack and each layer allows for boards to be easily removed or added to the system. With the current setup additional switches or power supplies can be added to the system and up to 92 boards can be supported. The completed rack is shown in Figure 3.3. Currently only the top half of the rack is used due to the lack of a second switch. With a second switch the bottom half can be used to support another 48 boards.

The cluster is also highly portable and can be powered entirely using one normal 110 Volt, 15 Amp wall socket. Every board is secured to their layer using screws and wires that are taped down to prevent movement when the cluster is being transported.

\subsubsection{Hardware Network Setup}

The server rack currently supports multiple switches that can be chained together depending on the use case of the cluster. Multiple 48 port switches are available and every port is able to reach up to 46 nodes using 12 foot Ethernet cables. 


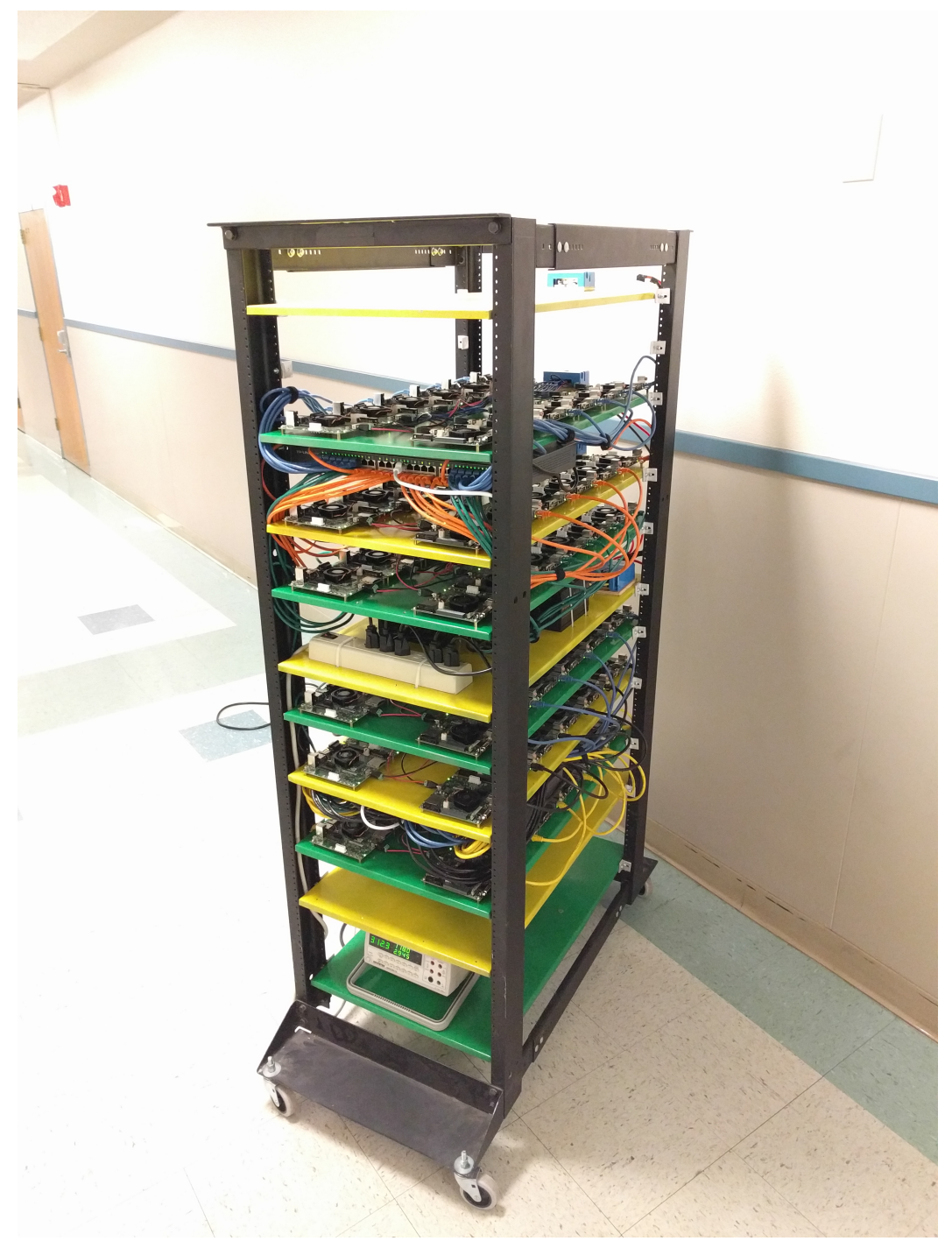

Figure 3.3: Completed Server Rack (46 nodes) 
The entire cluster uses Ethernet cables as opposed to fiber which most top supercomputers use. This is due to the high cost of fiber cables. While the performance increase of using fiber would be significant, the costs would be too high and Ethernet provides high enough speeds for many of the applications that would be run on a low power cluster. The network switches are placed in their own layer and can support up to 4 layers of boards per switch assuming 48 port switches are used.

As seen in Figure 3.4, Ethernet cables are organized by color for each layer and pulled to the side to prevent cluttering. This setup allows faster debugging of the node by making it easier to find out which nodes are not responding to network queries.

Currently the switch used in the cluster is a managed 48 port switch that supports STACK mode. In this mode multiple switches can be connected together but function as if it were one switch greatly reducing the bottleneck and latency that would result from connecting two switches together using normal Ethernet. With the addition of another switch the size of the cluster can be easily expanded without an increase in communication time between nodes.

\subsubsection{Hardware Summary}

Table 3.1 shows all the hardware components used to construct the cluster. A total of 80 Jetson TK1 development boards were purchased but only 46 were used in the final cluster due to network limitations. The total cost of the cluster not counting unused TK1 boards is 7557 dollars and took around 40 hours to build.

Many of the components used in the Astro cluster could be upgraded for a more powerful cluster. Primarily a simple network upgrade could double the size of the cluster and reduce cluster communication times by a significant margin. However, with the current build, the Astro cluster is extremely low cost and still provides a highly efficient compute power. 


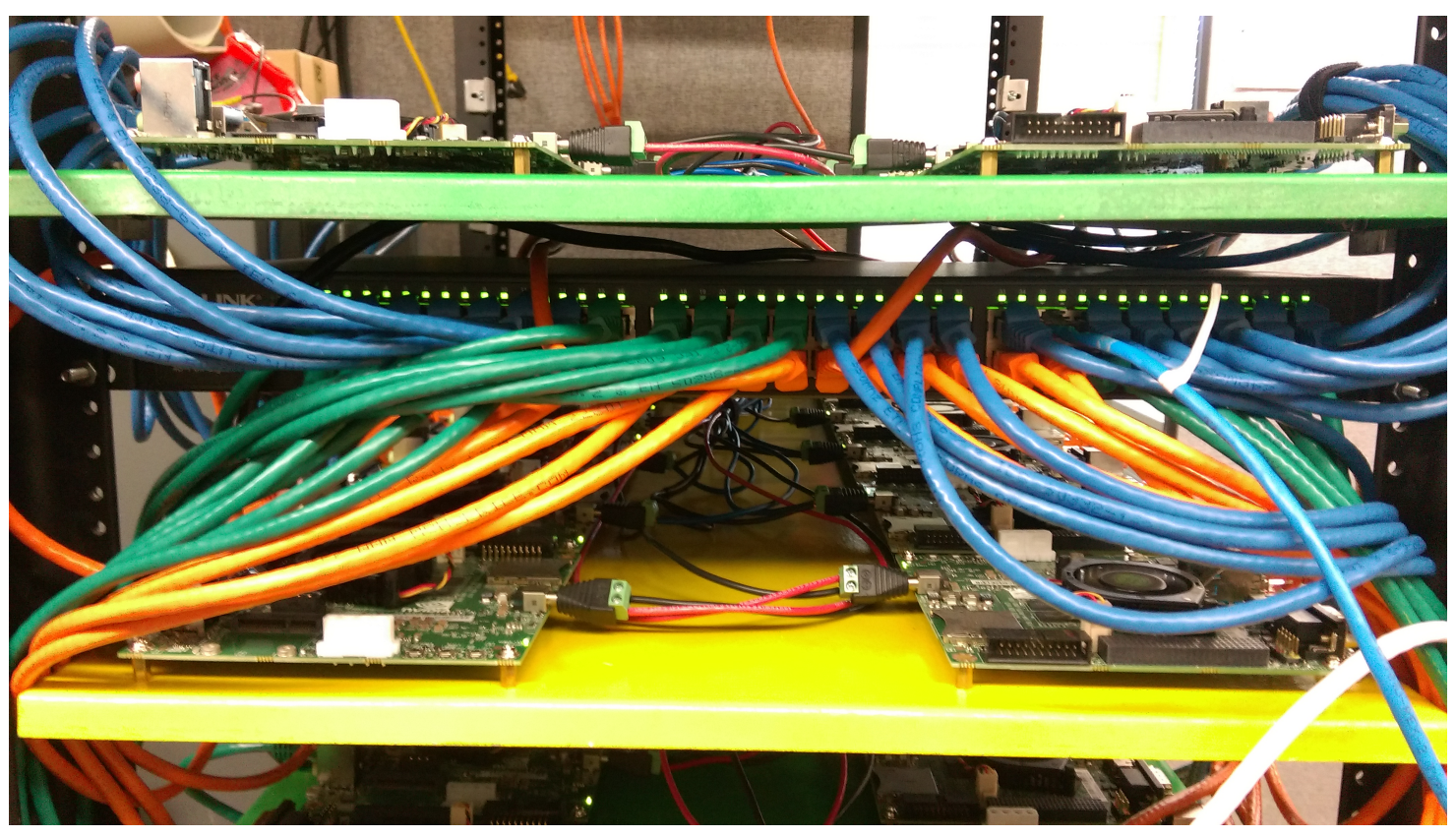

Figure 3.4: Switch setup for the Astro cluster

Table 3.1: Summary of Hardware components

\begin{tabular}{|c|c|c|c|}
\hline Hardware & Quantity & Individual Cost $(\$)$ & Total Cost $(\$)$ \\
\hline \hline Jetson TK1 & 80 & 135 & 10800 \\
\hline 48 Port Gigabit Switch & 1 & 500 & 500 \\
\hline 500W Power Supply & 2 & 310 & 620 \\
\hline Server Rack & 1 & 100 & 100 \\
\hline Cat6 Ethernet Cable & 48 & 1.50 & 12 \\
\hline Barrel Connector & 48 & 0.27 & 12 \\
\hline Copper Wire (8 Gauge) & $25 \mathrm{ft}$ & 12 & 20 \\
\hline Copper Wire (14 Gauge) & $200 \mathrm{ft}$ & 20 & 12147 \\
\hline Misc screws/bindings & 192 & 0.05 & \\
\hline Total & - & - & \\
\hline
\end{tabular}


Overall the cluster was simple to construct and relatively low cost for the amount of theoretical compute power available.

\subsection{Software}

Many of the software choices in the Astro cluster were made to meet the specified requirement but also to make sure the cluster runs as close as possible to real production clusters.

\subsubsection{Operating System}

The Linux4Tegra operating system [31] is used by all nodes in our cluster. This operating system is provided by NVIDIA specifically for the Jetson TK1 board and is a modification of Ubuntu. The operating system comes pre-installed on all boards and while alternatives are supported, many of the packages such as CUDA and MPI were supported by the OS and changing the OS was not necessary.

\subsubsection{Software Network Setup}

All nodes in the cluster are assigned an IP address, in the 10.0.0.0/24 subnet, based on their location starting with 10.0.0.1 in the top left corner of the uppermost rack. The nodes are than labeled from left to right incrementing the IP address. Using a static IP address based on location allows easier physical management of the nodes in case of hardware failure.

IP addresses are assigned using DHCP with a static IP table generated using the MAC-addresses of each node, the file used to generate the IPs can be found at appendix A. With the current IP scheme up to 255 boards are supported but a simple change of the subnet can easily increase the number of supported nodes in the cluster. 
SSH keys are distributed between all the boards allowing each board to connect to one another without using a password. This is done to allow MPI programs to communicate without having to specify a user and password for every connection.

To allow for easier installation a separate machine is connected to the system which has access to the Internet on a separate interface. The machine is forwarded all packets not in the 10.0.0.0/24 and forwards packets to and from the nodes in the cluster allowing for Internet access.

\subsubsection{Cluster Management}

A large component of operating a cluster is management of the cluster. Management of the cluster often includes two main components; managing installed libraries or applications, and monitoring system health.

\section{Managing Applications and Libraries}

In most commercial clusters tools such as ROCKS [24] are used to manage installed libraries and utilities. Tools like these handle keeping software versions synchronized and making sure all nodes are using the same libraries or applications. Unfortunately the Jetson TK1 does not support ROCKS due to lack of ARM support. Instead the Astro cluster heavily utilizes custom scripts and the distributed file system to keep track of installed libraries. All installations are performed on the main node including packages that need to be compiled from source. Once compiled and installed, the files are then transferred and copied to the local file system of each node using the distributed file system and simple bash scripts. The main node keeps track of all installed packages in a simple text file and confirms with each node that the installed packages match the versions mentioned in the file. While many of these packages can be used from the distributed file system without a full copy to the local file system, 
many applications such as Open MPI recommend a local copy for better performance.

While the management features are not ideal, the Astro cluster was not designed to be administered by many individuals and is instead a demonstration cluster. Simple scripts, and use of the distributed file system is enough to keep the system synchronized without complications. In a real production environment utilities such as ROCKS are necessary to make sure the system is stable and synchronized.

\section{Monitoring System Health and Stability}

When operating a cluster, it is important to keep track of overall system health and stability to ensure distributed applications are able to run. This involves making sure nodes are still alive as well as making sure temperatures and CPU utilization does not exceed expected amounts. Ganglia [21], a commonly used monitoring system for clusters, was used for system health monitoring.

Ganglia is extremely lightweight and sends a few packets at a configurable rate to gather statistics from nodes. For the Astro cluster we used a separate computer not involved in normal cluster operations to gather this data and display the data using the Ganglia web-frontend. By moving the statistical analysis to a separate machine, Ganglia has a minimal effect on normal cluster operations.

Ganglia is able to provide a large amount of information that can be configured for specific needs. For the Astro cluster, additional plugins to include overall cluster power usage and GPU usage were added, and unnecessary information (such as free disk space) was removed to reduce packet overhead. Individual statistics can also be polled at different rates, resulting in an extremely flexible and useful tool for cluster monitoring.

Figure 3.5 shows some of the cluster statistics Ganglia can display. Overall load of the entire cluster, active CPUs, overall network usage are all important for normal 


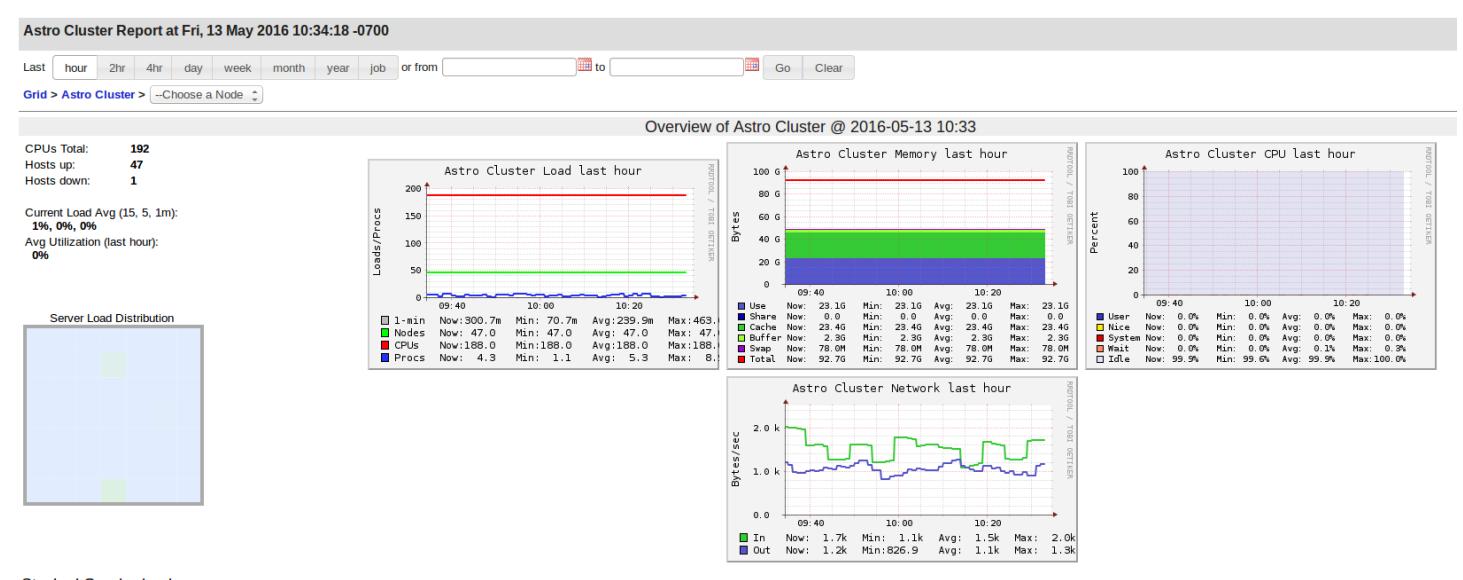

Stacked Graph - load_one

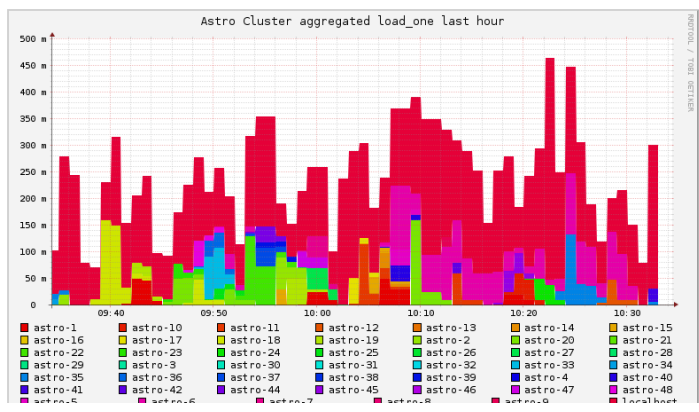

Figure 3.5: Ganglia web frontend main view

cluster operations and can be easily monitored using Ganglia. Ganglia also provides individual board statistics that are helpful for debugging or identifying inconsistent node behavior as shown in Figure 3.6.

Figure 3.7 shows the power meter used to monitor power usage in the cluster. The data shown on the meter is also available through a serial connection and the data read from serial is saved to a file and sent to Ganglia for easier monitoring.

Ganglia has two configuration files, one for each client (gmond.conf) and one for the main node collecting the data (gmetad.conf). The configuration for the main node uses the default configurations. Each client's configuration file is modified to send data to the main node and reduce overhead by removing some of the statistics being sent. The configuration file used by clients can be found in Appendix B. 


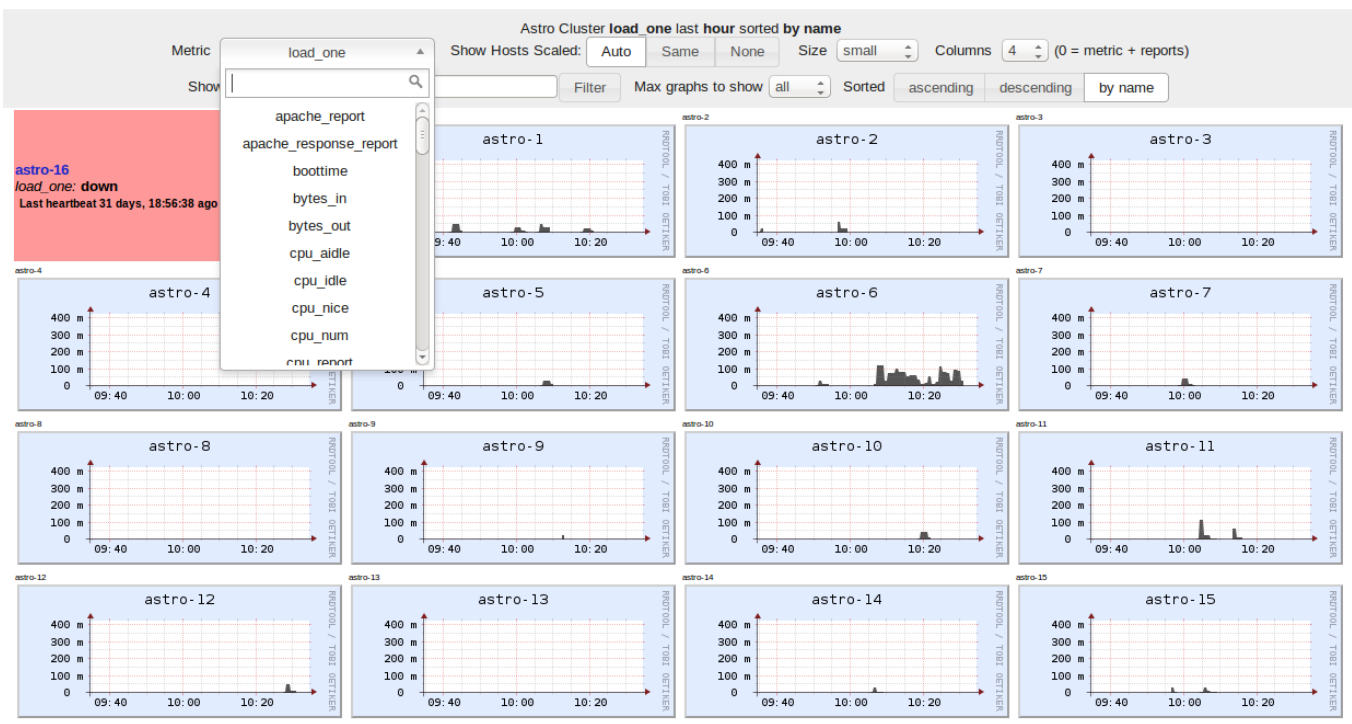

Figure 3.6: Ganglia web frontend alternate view

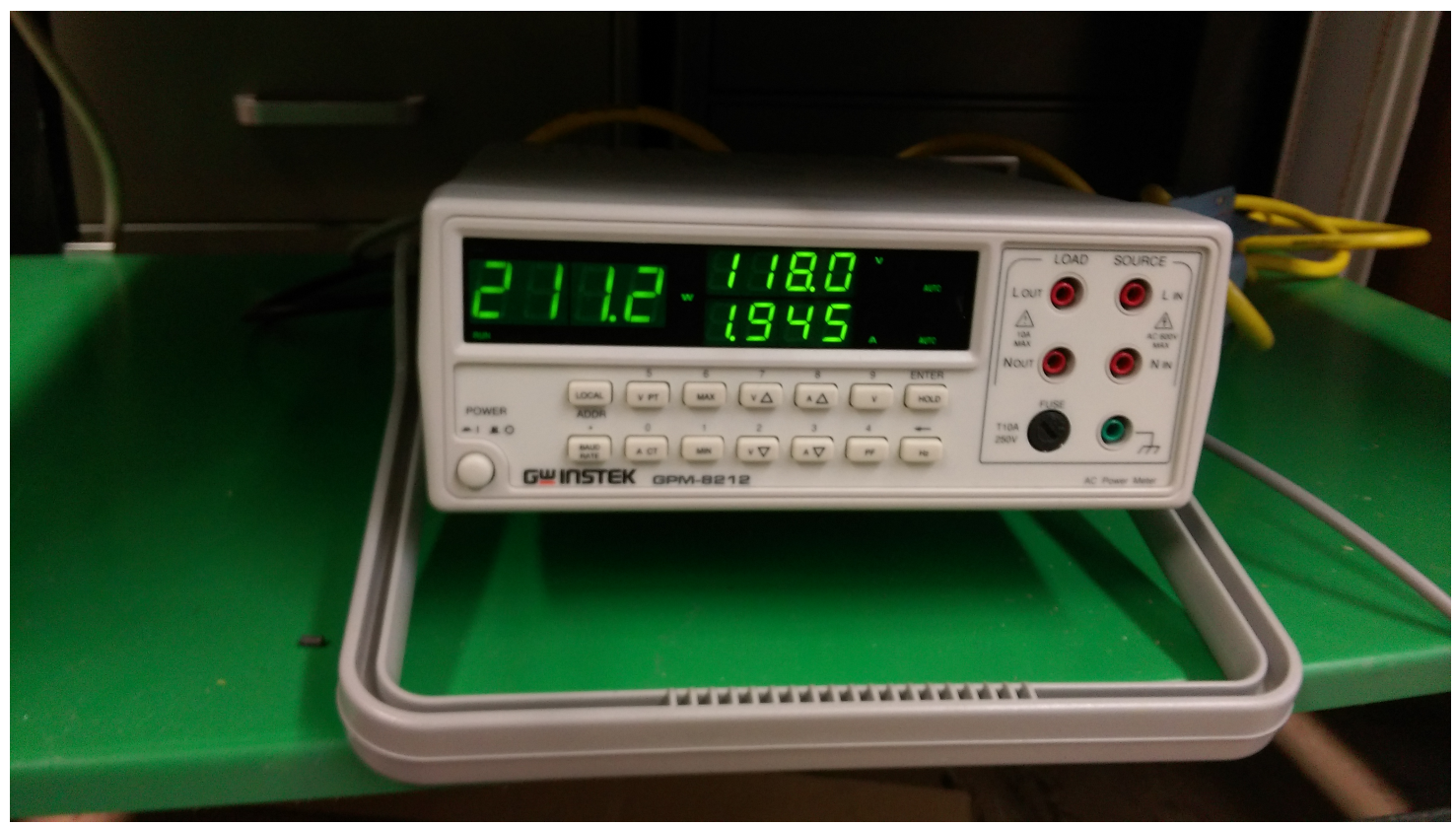

Figure 3.7: Power Meter used to monitor power usage of the cluster 


\subsubsection{Installed Packages and Libraries}

Before running benchmarks on our system, some critical packages are required: CUDA, MPI, and a BLAS (basic linear algebra subroutines) library. For CUDA, all boards are running CUDA 6.5 which at the time of this writing is the newest version available for the Jetson TK1. MPICH2 [5] and Open MPI [7] are both used for our message passing interface, and OpenBLAS [6] combined with cuBLAS [30] are used as the BLAS libraries.

These libraries are all compiled and installed on each node. Due to the homogeneous nature of the Astro cluster, compilation only needs to occur on one node.

Many of these packages were chosen due to system compatibility. Some of the faster BLAS libraries such as MKL are not supported by the Jetson TK1 due to lack of ARM support and could not be used.

\subsubsection{File system Installation and Configuration}

The configurations for the file systems used in the cluster are described in this section. OrangeFS and CephFS are relatively new file systems that still receive frequent updates, installation and configuration procedures may be different depending on the version being installed. The latest stable versions available at the time were installed in the cluster. Multiple file systems were installed to show the types of file systmes supported by the Astro Cluster

\section{NFS}

NFS is a common file system available by default on almost all Linux distributions. For the TK1, the option to support running a NFS server had to be enabled in the kernel. Only the main server needed this modification and clients could be installed 
with no modifications.

All packages were obtained using the package manager available on the TK1 (apt-get) and a single folder is shared between all nodes with the following configuration.

/home/astro/Desktop/astro 10.0.0.0/24(rw, sync,no_subtree_check)

\section{OrangeFS v2.9}

OrangeFS was installed following the instructions provided at [8]. FUSE is used as the main client to the file system interface due to the OrangeFS kernel being incompatible with the TK1 at the time.

OrangeFS is configured without security and a basic setup to support only directory sharing between all nodes. Four nodes are set up as servers within the cluster and the rest of the nodes act as clients. The servers are responsible for handling metadata and I/O within the cluster. By splitting up the workload between four nodes, files can be transmitted with less bottlenecks to the rest of the cluster.

\section{Ceph (INFERNALIS)}

Ceph was installed following the instruction provided at [1]. Similar to OrangeFS, FUSE is used and a minimally configured Ceph is installed.

CephFS is configured with one admin node, four metadata nodes, two monitoring nodes, and every node acting as an object storage device. The object store is specified to be a directory, and the directory is accessible by all nodes. The metadata nodes handle metadata associated with these files while the monitoring nodes keep track of file system health and functionality. The number of metadata, monitor, and storage nodes can be easily modified while the file system is running using the admin node. 


\subsection{Modifications to High Performance Linpack}

Modifications were made to the CUDA-enabled HPL benchmark provided by NVIDIA (HPL-2.0-FERMI). The benchmark is designed for FERMI-class cards and without modification is incompatible with the Kepler GPU used by the TK1.

\subsubsection{Removing Incompatible CUDA Functions}

The Jetson TK1 is a 32-bit system and is unable to support some of the 64-bit specific functions provided by the CUDA library. The functions are shown below.

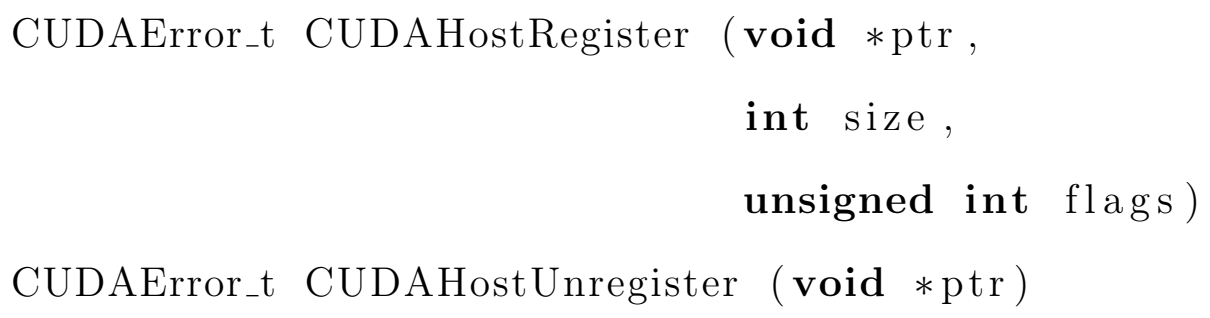

The original HPL benchmark uses CUDAHostRegister, which allows certain ranges of memory to be page-locked allowing faster data transfers between the CPU and the GPU, but is unavailable for 32-bit systems. Since the TK1 is able to share memory between the CPU and the GPU, this function was removed and replaced with zerocopy mechanisms using the functions below.

CUDAError_t CUDAHostAlloc (void **pHost, int size, unsigned int flags )

CUDAError_t CUDAFreeHost (void $*$ ptr)

CUDAHostAlloc has similar functionality as CUDAHostRegister and pins the memory for faster memory transfers. For the TK1, the memory is marked and can be used freely between the CPU and GPU without tranfers. CUDAFreeHost cleans up the memory region and unpins it similar to the CUDAHostUnregister function. 
Table 3.2: NVIDIA profiler summary for HPL with memcpy

\begin{tabular}{|c|c|c|c|}
\hline CUDA Function & Time (\%) & Time & Calls \\
\hline \hline cuMemcpy2DAsync & $75.60 \%$ & $3.735 \mathrm{~s}$ & 303 \\
\hline cudaFree & $21.11 \%$ & $1.043 \mathrm{~s}$ & 312 \\
\hline cudaEventSynchronize & $0.02 \%$ & $1.0398 \mathrm{~ms}$ & 37 \\
\hline
\end{tabular}

The CUDA enabled HPL benchmark is also designed to be used by FERMI class GPUs and utilizes an optimized version of the $d g e m m$ function. Since these functions can not be used by the TK1, unoptimized general versions of the function from the cuBLAS libraries are used instead. No optimizations were made to these cuBLAS functions for the TK1.

\subsubsection{Modifying Memory Usage}

The optimizations made to the original HPL benchmark also uses memory sizes that are too large for the Jetson TK1. Scratch size and GPU memory allocation values were modified to support the actual amount of space available on the TK1.

The original benchmark was also written for 64-bit systems and many of the values used assumed 64-bit size_t sizes. Changes were made to make sure values fall within the correct range and were modified for 32-bit systems.

Memory allocations were also changed to take advantage of zero-copy mechanisms available on the TK1. While the original HPL benchmark takes advantage of pinned memory to streamline the memory transfer process, the TK1 is able to completely remove this step and pass a pointer instead. Using the nvprof [32] utility, the time savings of removing the memory transfer times can be easily seen.

Table 3.2 shows the timings of specific CUDA functions before modifications to 
Table 3.3: NVIDIA profiler summary for HPL with zero-copy

\begin{tabular}{|c|c|c|c|}
\hline CUDA Function & Time (\%) & Time & Calls \\
\hline \hline cudaEventSynchronize & $80.05 \%$ & $3.42 \mathrm{~s}$ & 37 \\
\hline cudaFree & $8.05 \%$ & $856 \mathrm{~ms}$ & 312 \\
\hline cudaHostAlloc & $0.09 \%$ & $1.058 \mathrm{~s}$ & 1 \\
\hline
\end{tabular}

take advantage of the TK1. Specifically the cuMemcpy2DAsync function takes a total of 3.735 seconds, which is completely removed when running the modified version as shown in Table 3.3. The replacement function cudaHostAlloc removes the need for the memcpy and only takes 1 second. It is important to note that a single call of cudaHostAlloc takes 1.058 second while 303 calls of cuMemcpy2DAsync took around 3.735 seconds. Smaller problem sizes may still run faster using the normal memcpy method depending on the number of memcpy calls required.

While the modified version does remove the memory transfer times, it spends a longer time to synchronize all the threads and results in a similar overall run-time. The cudaEventSynchronize function takes a significantly longer portion of time in the zero-copy version and most likely results from unoptimized synchronizes that occurs due to memory allocation changes.

While the modifications did not dramatically speed up the overall run-time of the program, with proper optimization and changes to the synchronization phase of the benchmark, improvements can likely be seen. The removal of memory transfers removed the cuMemcpy2DAsync call entirely which can be a signifiant portion of the run-time of the program. 


\subsubsection{Configuring the HPL benchmark}

Before running the HPL benchmark, an input file is required that specifies a few parameters needed to run the benchmark. The configuration parameters modified for the run used for the Astro cluster are problem size, matrix orientation, and block size. These values were changed to the recommended values for the memory available per board, size of the cluster, and cache sizes with some minor trial and error changes used to obtain the fastest performance available for each node.

Both the CPU and GPU were also used simultaneously when running the GPU version of the benchmark. The original source code allowed for problems to be split between the CPU and GPU resulting in greater parallelism as long as an optimal split value is used.

The amount of work split between the CPU and GPU was also determined using a trial and error method and the NVIDIA profiler to determine the best split. The optimal split occurs when both the CPU and GPU finish their assigned task at the same time so no blocking due to synchronization is required. This split changes depending on the number of nodes and the problem size, but a $85 \%$ GPU and $15 \%$ CPU split was generally optimal for all problem sizes. Minor adjustments to these split values are also automatically determined by the benchmark to find the nearest optimal split during run-time.

\subsection{Modifying the LAMMPS Benchmark}

The LAMMPS benchmark had minimal modifications when compiled from source. To take advantage of the multiple core CPU, the threaded plugin provided as part of the source for LAMMPS was installed and 4 cores were used. 


\subsubsection{Configuring the LAMMPS Benchmark}

Similar to the HPL benchmark, LAMMPS also requires an input file that specifies the type and number of atoms, and the type of simulation. For the tests used in our benchmark, a Lennard-Jones fluid simulation is used due to the high communication between nodes required for the benchmark. The input file problem size is scaled with the number of nodes, and a base size was determined by choosing a size that results in a minimal 15 second run-time. The GPU version of the benchmark also takes advantage of the CPU for some setup, but performs all the calculations on the GPU and no split of work needs to be configured.

\subsection{Shortcomings of the System}

While the cluster intends to emulate a real world HPC cluster, certain sacrifices were made due to cost or incompatibility with the Jetson TK1.

\subsubsection{Limited File System Support}

A few file systems were tested on the Astro cluster, but the most common file system Lustre was not tested. As mentioned previously Lustre is incompatible with the TK1 and could not be tested in the cluster.

Many of the tested file systems also had complications when attempting to use the kernel-space implementations. While kernel modules generally installed without error, the file system was often unstable and needed to be reinitialized. Some of this could be attributed to the default kernel available in the TK1, but no work has been done to explore using a different kernel distribution.

The Astro cluster was also relatively small compared to most production clusters,

and file system scalability was difficult to test. Many of the file systems' features such 
as data replication and faster I/O were unable to be fully tested until a larger cluster is available.

\subsubsection{Performance Tools}

Many of the performance measuring tools available to other systems were also unable to be used on the Jetson TK1. NVIDIA-SMI, a tool used to monitor GPU usage, is unavailable on the TK1 and GPU usage had to be estimated.

BLAS library choices were also limited by the Jetson TK1 architecture. The top BLAS library, MKL, is not supported by the ARM architecture. Luckily BLAS libraries were not crucial for many of the performance benchmarks due to the use of cuBLAS for the majority of BLAS operations.

\subsubsection{Network}

The network of the Astro cluster uses a single managed Gigabit switch and Cat-6 Ethernet cables. While the speeds provided by the network equipment are enough for basic validation and benchmarking, faster network equipment is common in production clusters. For our testing purposes the speed of the switch was enough to produce good benchmark results.

The biggest downside of the network setup was the lack of a second switch. With a second switch the cluster could be scaled to double the size allowing for better scalability tests for the benchmarks and file systems. 
Chapter 4

RESULTS

This chapter details the experimental setup and validation for the Astro cluster.

\subsection{Experimental Setup}

Prior to running the benchmarks, a few components in the cluster have to be properly configured. The Cluster setup, measurement tools, and testing environment are detailed below.

\subsubsection{Cluster Setup}

All benchmarks are tested on 46 identical Jetson TK1 nodes running the same operating system, file system, and libraries. All nodes have all CPU cores enabled and any power saving functionality is disabled. CPU and GPU have clock-speeds set to maximum.

Before running any benchmark, Ganglia is used to confirm the health of all nodes. Nodes are expected to be using anywhere from 0-5\% CPU, have most of their memory available, and be stable for at least 30 minutes.

\subsubsection{Measurement Tools}

The power meter and temperature meters are setup to feed into an external computer that keeps track of the data and provides a visualization through Ganglia. An external computer is used to prevent any interference between the measurement tools and the benchmarks being run. CPU measurement is performed through Ganglia and is 
logged every second. GPU measurements are unavailable for the system, and instead are estimated through power increase in the system as GPU tasks increase.

\subsection{Power and Temperature}

The power and temperature meters log data every second and save results to a file. The results in this section use the average power and temperature over 10 runs.

CPU utilization was set using stress [9], a UNIX utility that can set CPU and memory usage, while GPU utilization was estimated using benchmarking utilities,due to the lack of NVIDIA-SMI for the Jetson TK1.

While idle, the entire 46-node cluster including network equipment uses around 200 Watts. When all CPU cores and the GPU are fully utilized the cluster uses around 560 Watts.

Three power benchmarks were performed on the cluster: a CPU only test, GPU only, and a combination of both. Due to the lack of NVIDIA-SMI, the GPU power usage numbers are estimated using scaling GPU benchmarks. Figure 4.1 shows the power usage as utilization of the cluster increases.

The power usage of the cluster scales very well with utilization with a slight spike at around $25 \%$ utilization for the CPU. This is expected and most likely occurs due to the activation of CPU cores when CPU utilization starts maxing out a single core out of the four available on the CPU. Network and other devices on the nodes are not used for these power measurements and peak power usage will be higher depending on the amount of network communication and memory usage.

This power benchmark shows the advantages of using a GPU due to its lower power usage. Assuming the GPU can produce more FLOPS then the CPU, the GPU will also be significantly more power efficient and ideal for low power clusters. 


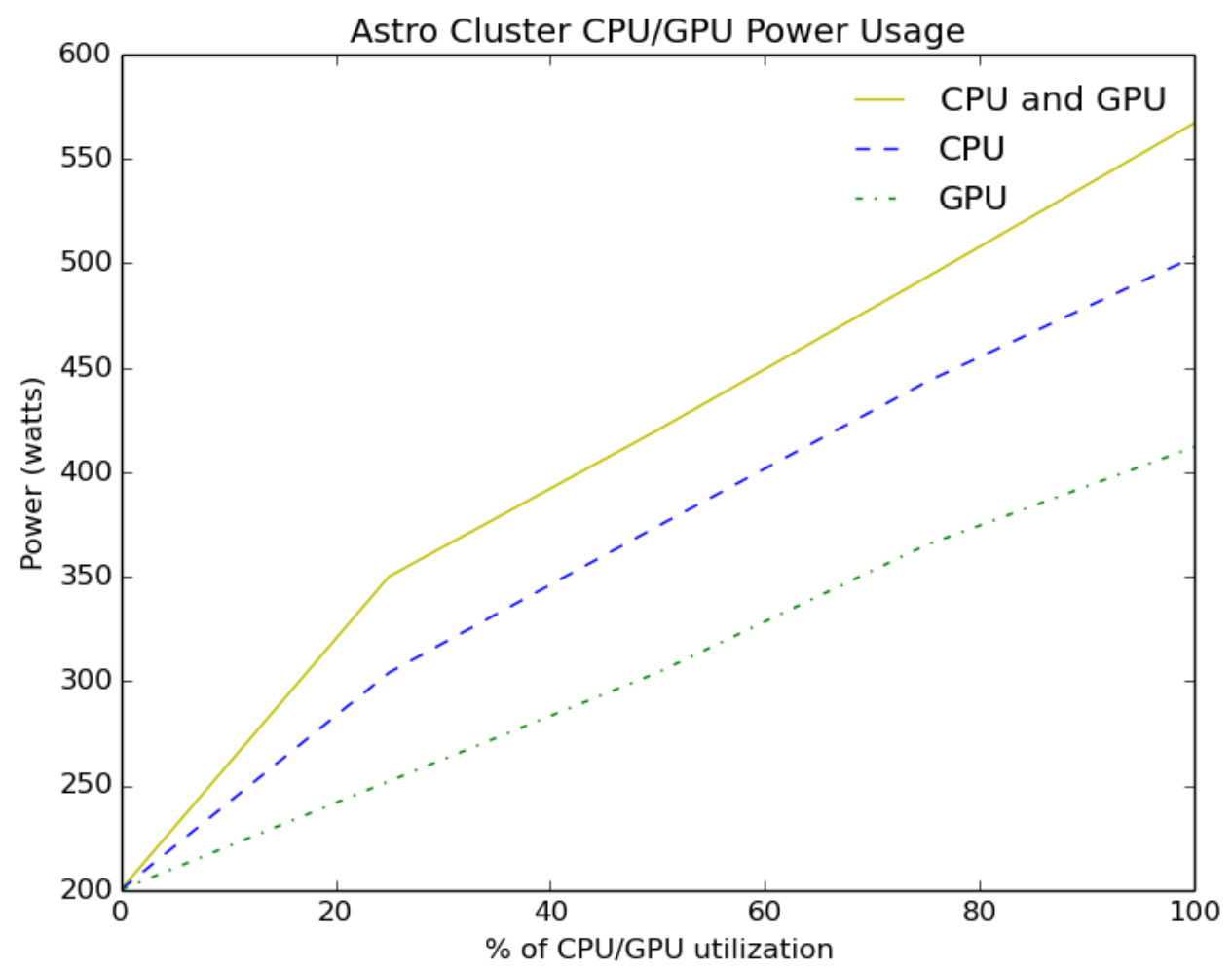

Figure 4.1: Power usage of the cluster as utilization increases 
The Jetson TK1 boards are cooled by a single fan with one speed setting. No major overall temperature increases were recorded while running the above power tests even at peak utilization. The low overall power usage and the fan are able to keep the cluster within half a degree Celsius of normal idle temperature.

\subsection{Network}

The cluster uses normal Gigabit Ethernet for inter-node communication. Using iPerf and 1 gigabyte file sizes, the bandwidth of the system is measured and shown in Figure 4.2. For this test, one node is elected as the server node and all other nodes are clients that try to download a file from the server. As the number of connections scales up, the overall bandwidth does not decrease by a significant margin, and is relatively close to the theoretical limit of 1000 Mbit. This is expected, since the

Astro cluster currently uses a single switch and does not have enough devices to bottleneck the switch.

The latency of the system was also low, and averaged at around $0.5 \mathrm{~ms}$ between any two nodes.

\subsection{Memory Bandwidth}

The memory bandwidth of the system is benchmarked with STREAM running with 2 million elements. The results of the benchmark for a single node can be seen below. The specific operations relate to common I/O operations for different types of applications. Copy tests copying data between memory locations, scale copies than multiplies the memory, add adds to the copied memory, and triad is a combination of all three. 


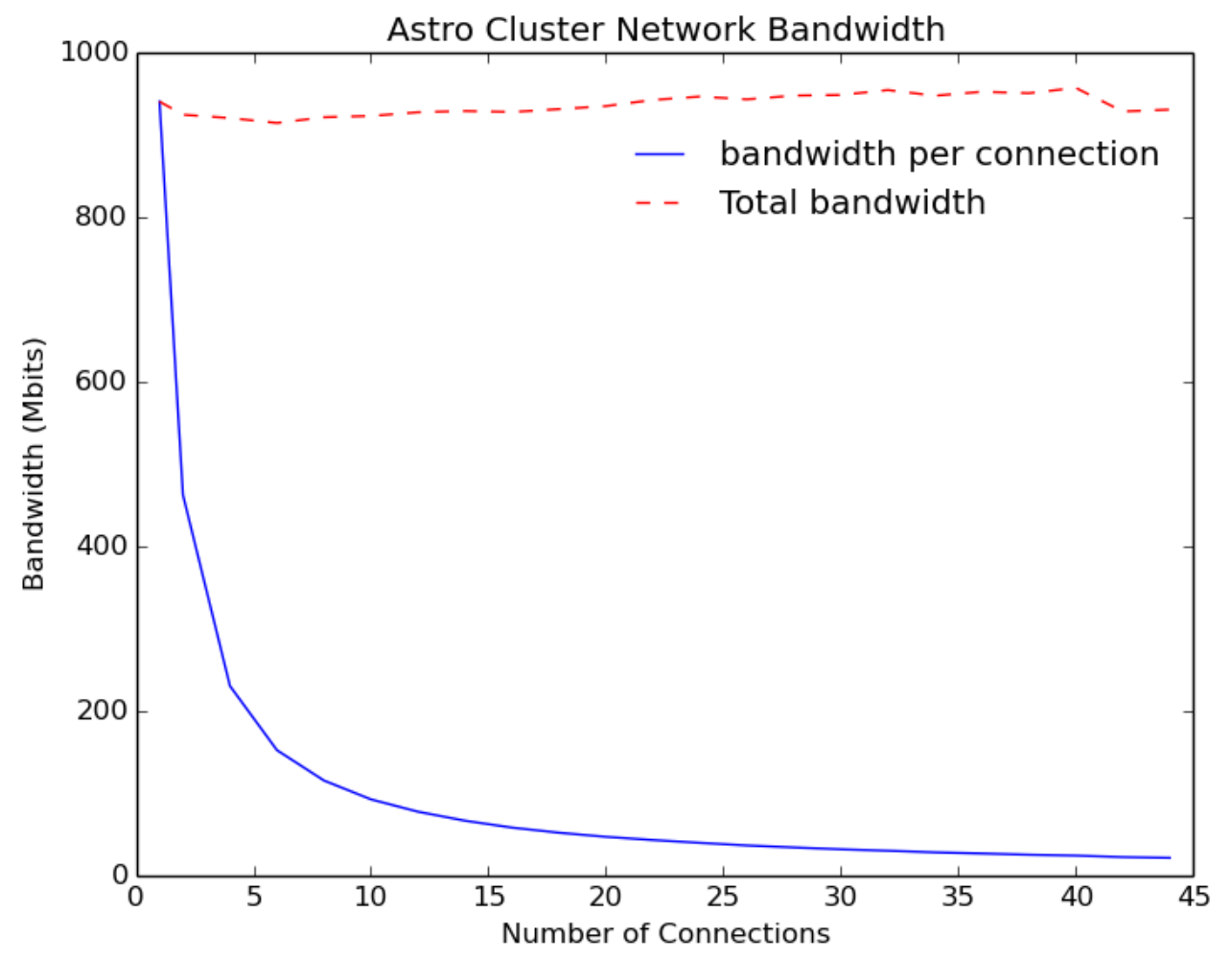

Figure 4.2: Network Bandwidth in the Astro cluster 


\begin{tabular}{|c|c|c|c|}
\hline copy & scale & add & triad \\
$3440 \mathrm{MB} / \mathrm{s}$ & $3410 \mathrm{MB} / \mathrm{s}$ & $3683 \mathrm{MB} / \mathrm{s}$ & $3686 \mathrm{MB} / \mathrm{s}$ \\
\hline
\end{tabular}

The Jetson TK1 performs well in terms of memory bandwidth and is about twice as fast as a Raspberry Pi 2 [22]. The benchmark performs best with 4 OMP threads and averages to around $3500 \mathrm{MB} / \mathrm{s}$ for all operations.

\subsection{Single-Node Performance}

In this section we discuss the performance of a single Jetson TK1 board. We first benchmarked a single node using some of the sample code provided by the CUDA libraries. While running the N-body simulation provided in the CUDA SDK, a single board was able to achieve around 190 GFLOPS of performance as advertised.

Next we benchmarked a single node using HPL (high-performance LINPACK) using CPU- and GPU-only implementations. We compiled and ran the benchmark using OpenBLAS as our BLAS library, and Open MPI as our MPI library. Both CPU and GPU benchmarks were run using the NVIDIA provided CUDA enabled HPL. To test a CPU-only run, the variables that determine how much work is split between the CPU and GPU were set to 0, resulting in $100 \%$ of the work being designated to the CPU. For a single node, both CPU and GPU benchmarks resulted in 14 GFLOPS of performance with a problem size of 10240 .

To understand why the HPL benchmark using the GPU was under-performing, we analyzed the CUDA computations in the HPL benchmark. HPL aims to solve a dense linear system and utilizes the dgemm function in the cuBLAS library, which when benchmarked individually resulted in around 14 GFLOPS of performance. Other similar functions such as cgemm were able to reach 161.52 GFLOPS of performance as expected. These measurements match those listed for this specific GPU architec- 
ture [32] which shows the single to double precision ratio of the Jetson TK1 is around 1:24. This means that double precision speed should be around $1 / 24$ th the speed of single precision.

Some of the commonly used LAPACK routines were benchmarked and the results are shown in Figure 4.3. The sgemm and cgemm functions are single-precision operations while dgemm and zgemm are double-precision operations. All results are the peak possible performance seen over 10 runs of the benchmark. CPU performance was measured using 4 OMP threads to take advantage of all available CPU cores. For single precision operations, we can see that the GPU greatly outperforms the CPU and is able to reach a maximum of around 211.17 GFLOPS. Double precision operations performed equally slowly on both the CPU and the GPU, but are able to reach the theoretical max of around 14 GFLOPS on the TK1, which explains the results of the HPL benchmark.

\subsection{Cluster Performance}

The Astro cluster is benchmarked using 46 devices with all CPU and GPU speeds set to max.

Figure 4.5 shows the performance of the cluster as the number of nodes increases. Results are averaged over 10 runs. Problem sizes and input file configurations for each node count are adjusted to obtain the maximum performance, and usually involved using up as much memory as possible on each node. The exact configuration variables can be seen in Table 4.1. P and Q refer to matrix dimensions used by the benchmark. An example input file is shown in Figure 4.4. Both CPU and GPU implementations resulted in very similar performance numbers and scaled well with the number of nodes involved in the benchmark. The peak performance of the cluster resulted in around 326 GFLOPS of performance at 44 nodes, which is $52 \%$ of the theoretical 


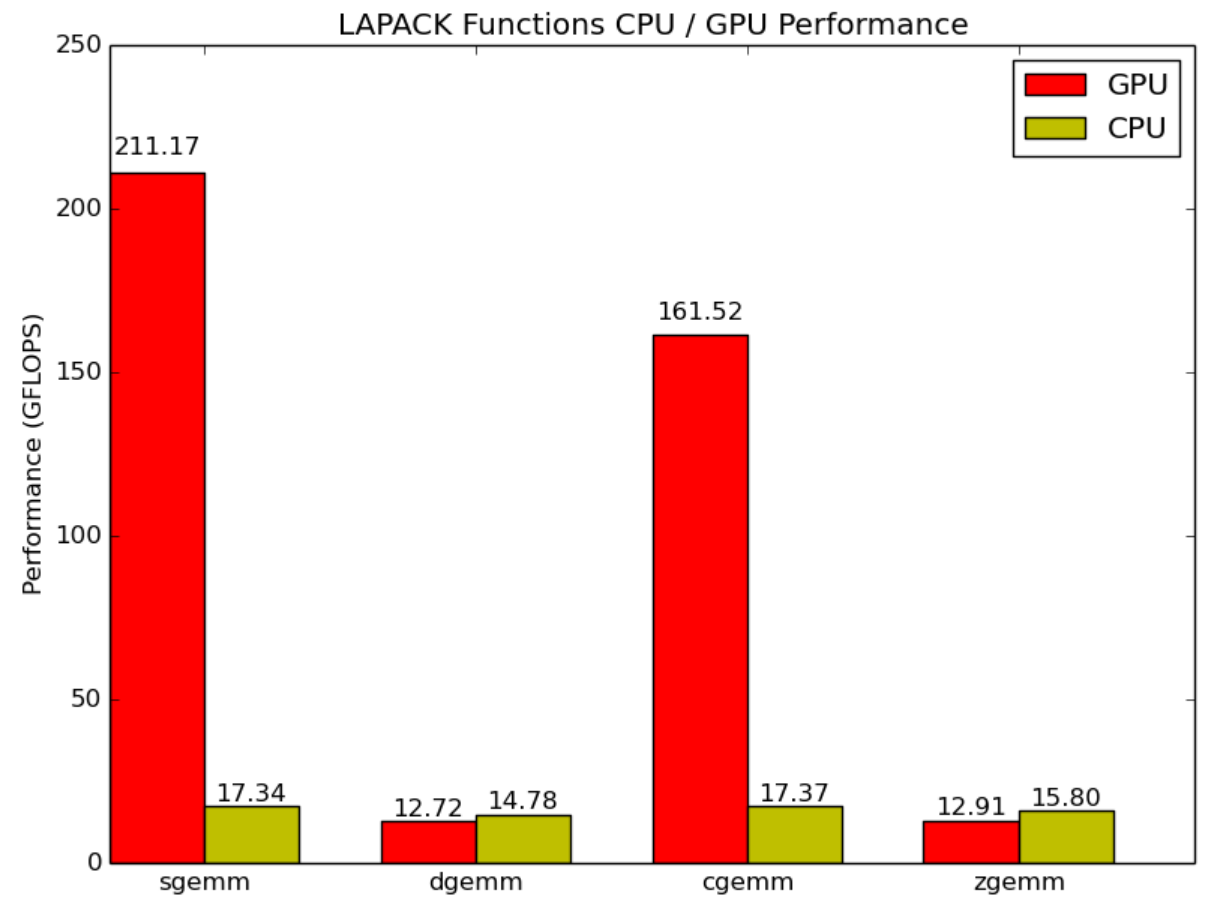

Figure 4.3: Performance of various LAPACK routines for CPU/GPU 
Table 4.1: Input Values for HPL benchmark

\begin{tabular}{|c|c|c|c|}
\hline Number of Nodes & Problem Size (N) & P & Q \\
\hline \hline 1 & 7168 & 1 & 1 \\
\hline 2 & 10240 & 1 & 2 \\
\hline 4 & 14336 & 2 & 2 \\
\hline 8 & 20480 & 2 & 4 \\
\hline 12 & 22528 & 3 & 4 \\
\hline 16 & 24576 & 4 & 4 \\
\hline 20 & 26624 & 4 & 5 \\
\hline 24 & 28672 & 4 & 6 \\
\hline 28 & 30720 & 4 & 7 \\
\hline 32 & 32768 & 4 & 8 \\
\hline 36 & 33792 & 6 & 6 \\
\hline 40 & 35840 & 5 & 8 \\
\hline 44 & 37888 & 4 & 11 \\
\hline 46 & 39936 & 2 & 23 \\
\hline
\end{tabular}

peak performance of 44 Jetson TK1's and is reasonable due to communication and distribution overhead. The slower speeds for the 2 and 46 node experiment are likely due to a bad matrix split and to the difficulty of creating an even matrix size for those node counts. While a single-precision HPL benchmark is unavailable, using the built in NVIDIA single-precision utilities, we were able to reach the expected singleprecision performance of around 6800 GFLOPS. This follows the same trend we see from the double-precision HPL results.

While the CPU and GPU had similar performance numbers, power usage varied greatly between the two implementations. Figure 4.6 shows that CPU power usage 


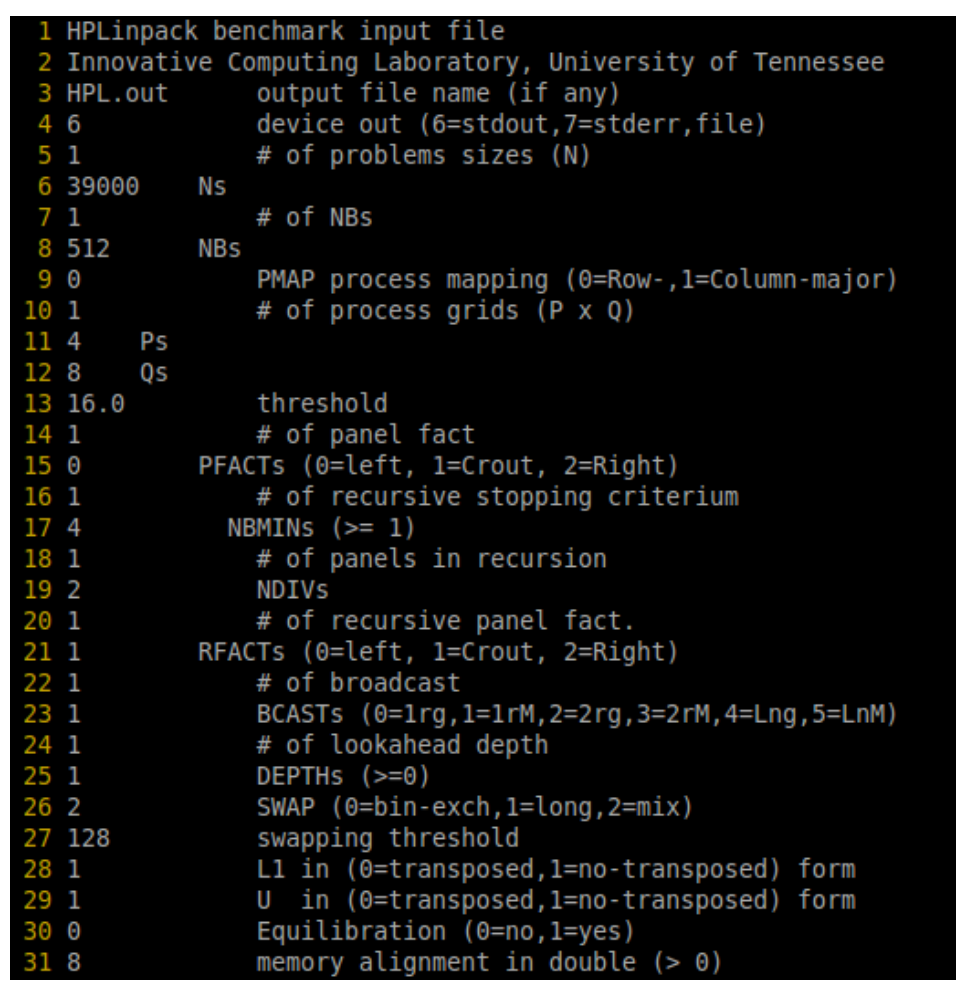

Figure 4.4: Example input file for HPL benchmark: only N, P, and Q are modified for different node sizes

is higher than GPU power usage by a large margin.

For both runs, the peak power usage is significantly higher than when testing power for the CPU and GPU alone. What is likely happening is that the communication between nodes and memory utilization contribute to the increased power usage.

Our next benchmark was aimed at measuring the parallel efficiency of the system and gaining some insight into how the network configuration may be impacting performance. The LAMMPS benchmark is a molecular dynamics simulator that reflects other classic HPC application codes. In our runs, the system is benchmarked with 125k atoms per node using the Lennard-Jones input with the problem size scaling with the number of nodes. The matrix split used for each problem size is shown in Table 4.2, problem sizes are automatically calculated by the benchmark, and depend on the number of nodes running the benchmark. Figure 4.7 shows an example input 


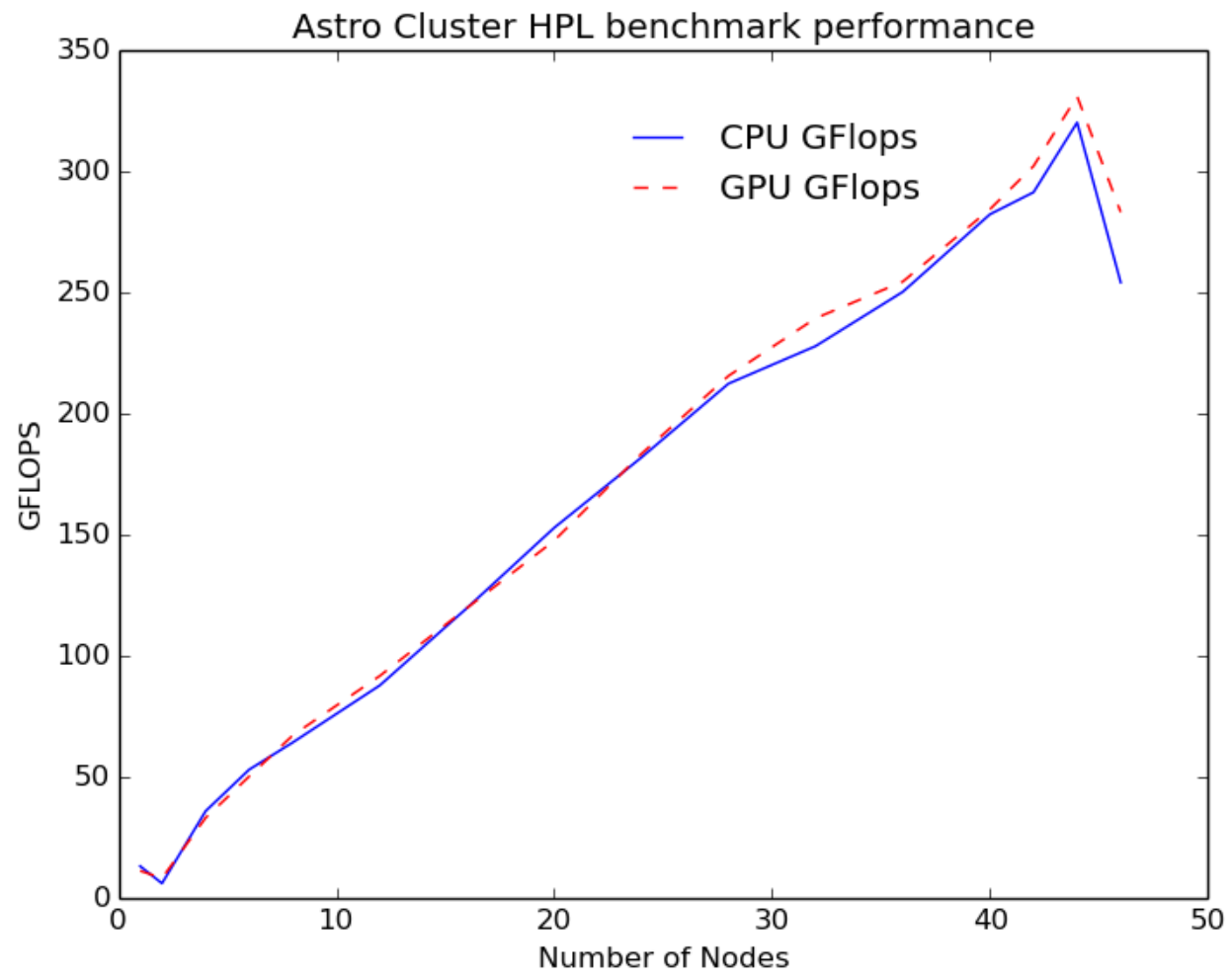

Figure 4.5: HPL benchmark scaling run with both CPU and GPU only implementations 


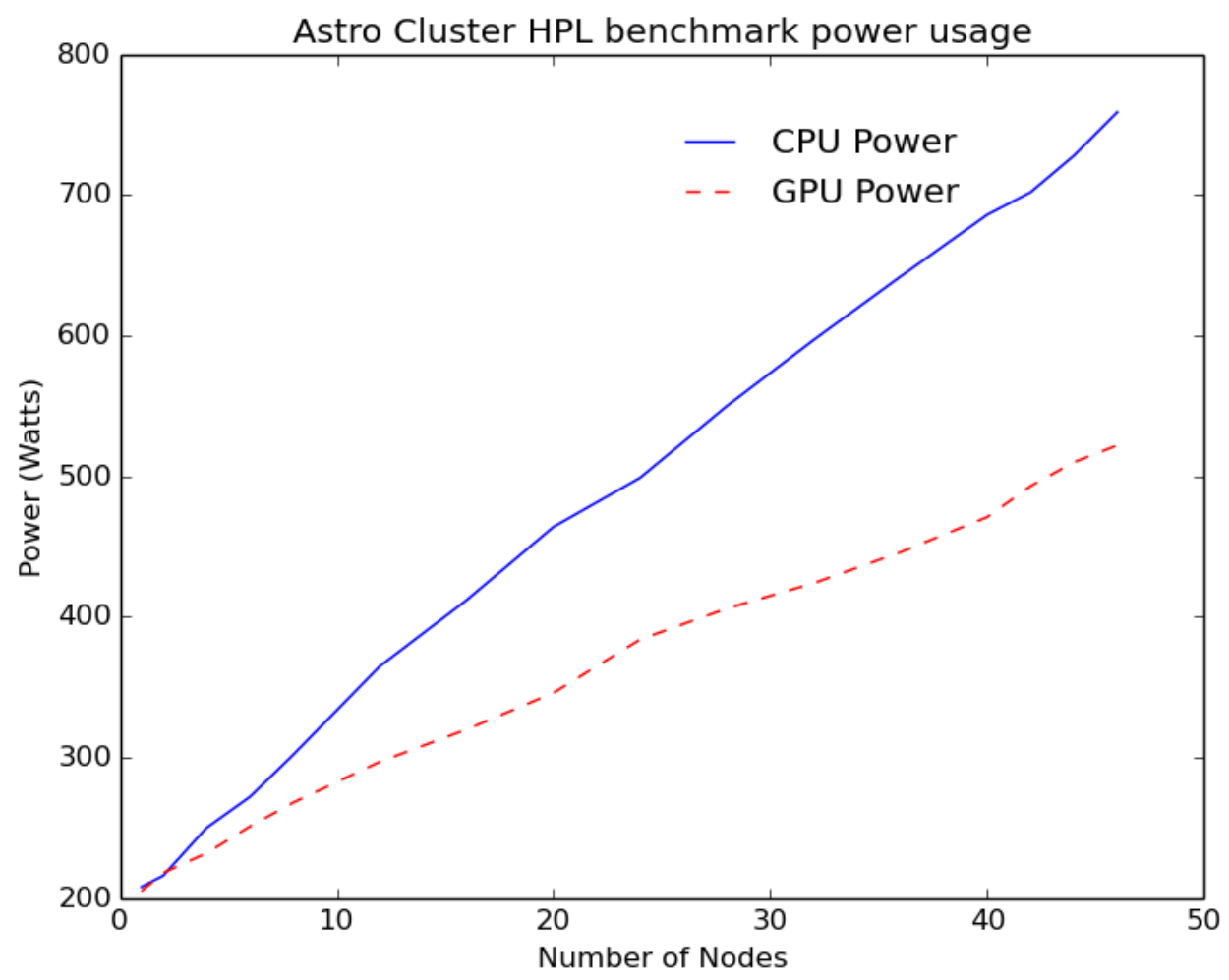

Figure 4.6: HPL benchmark power usage for both CPU and GPU 
Table 4.2: Input Values for LAMMPs benchmark

\begin{tabular}{|c|c|c|c|}
\hline Number of Nodes & X & Y & Z \\
\hline \hline 1 & 1 & 1 & 1 \\
\hline 2 & 1 & 1 & 2 \\
\hline 4 & 1 & 2 & 2 \\
\hline 8 & 2 & 2 & 2 \\
\hline 12 & 2 & 2 & 3 \\
\hline 16 & 2 & 2 & 4 \\
\hline 20 & 2 & 2 & 5 \\
\hline 24 & 2 & 3 & 4 \\
\hline 28 & 2 & 2 & 7 \\
\hline 32 & 2 & 4 & 4 \\
\hline 36 & 3 & 3 & 4 \\
\hline 40 & 2 & 4 & 5 \\
\hline 44 & 2 & 2 & 11 \\
\hline 46 & 1 & 2 & 23 \\
\hline
\end{tabular}

file with a slight modification to the base amount of atoms (variables $\mathrm{xx}, \mathrm{yy}$, and $\mathrm{zz}$ ) used for the benchmark. Each process uses a single GPU and corresponds to a single node. A CPU-only experiment was also run, but was significantly slower than the GPU. Figure 4.8 shows the results of a CPU vs GPU run.

The CPU implementation uses 4 OMP threads and the same problem sizes as the GPU version, but is around three times slower. Both the CPU and GPU runs show a similar trend with a slight increase in run time as we increase the number of nodes. In an ideal system the run time will be perfectly horizontal since the amount of work per machine stays the same as the problem size and number of nodes involved increases. 


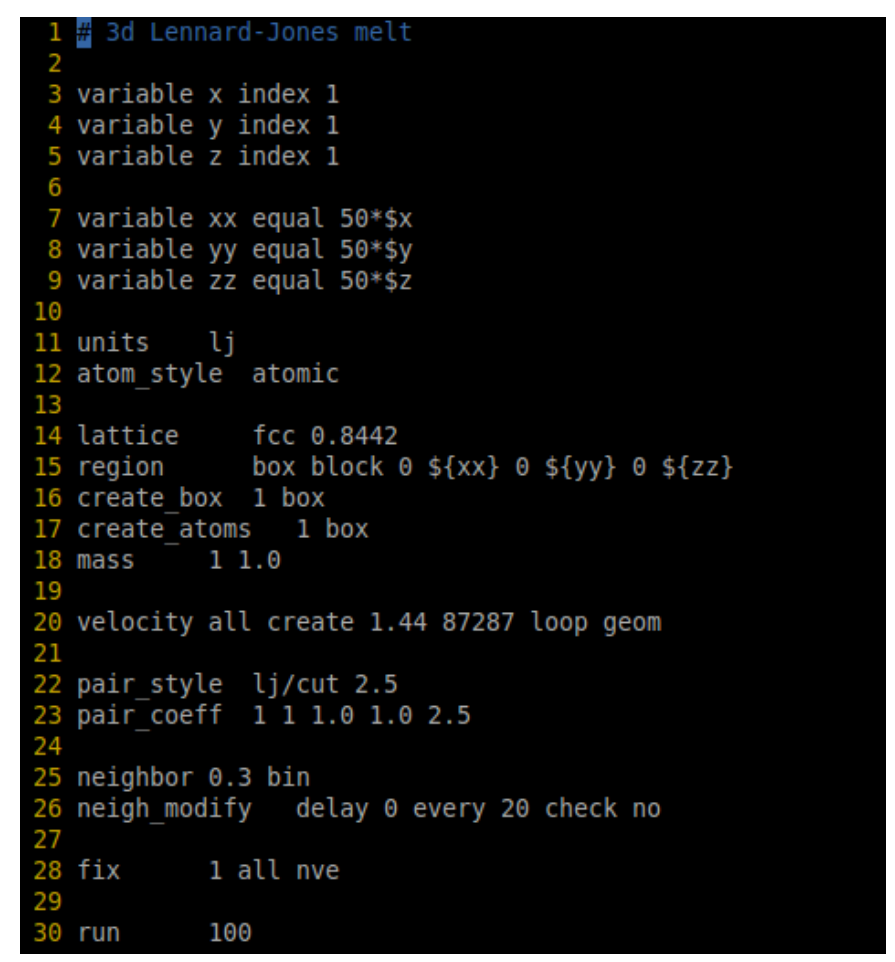

\section{Figure 4.7: Example input file for Lammps benchmark}

The following analysis is performed using the GPU benchmark due to its faster run times.

From Figure 4.9 we can see that communication time is the largest bottleneck and is responsible for the increase in total time. The rest of the calculations stay relatively horizontal and scale very well as we increase the number of nodes. This indicates that the cluster performs well when being scaled up. Increased communication time is unavoidable, but can be reduced with better network equipment.

When communication time is removed from the graph, we see that the total time stays almost entirely horizontal with a small increase at 46 nodes due to a difficult to split problem size. We expect a 48 node run will likely result in a better run time. All calculations scale well which shows that the biggest bottleneck in the cluster is node-to-node communication.

Using the data from the benchmark the parallel efficiency of the cluster is cal- 


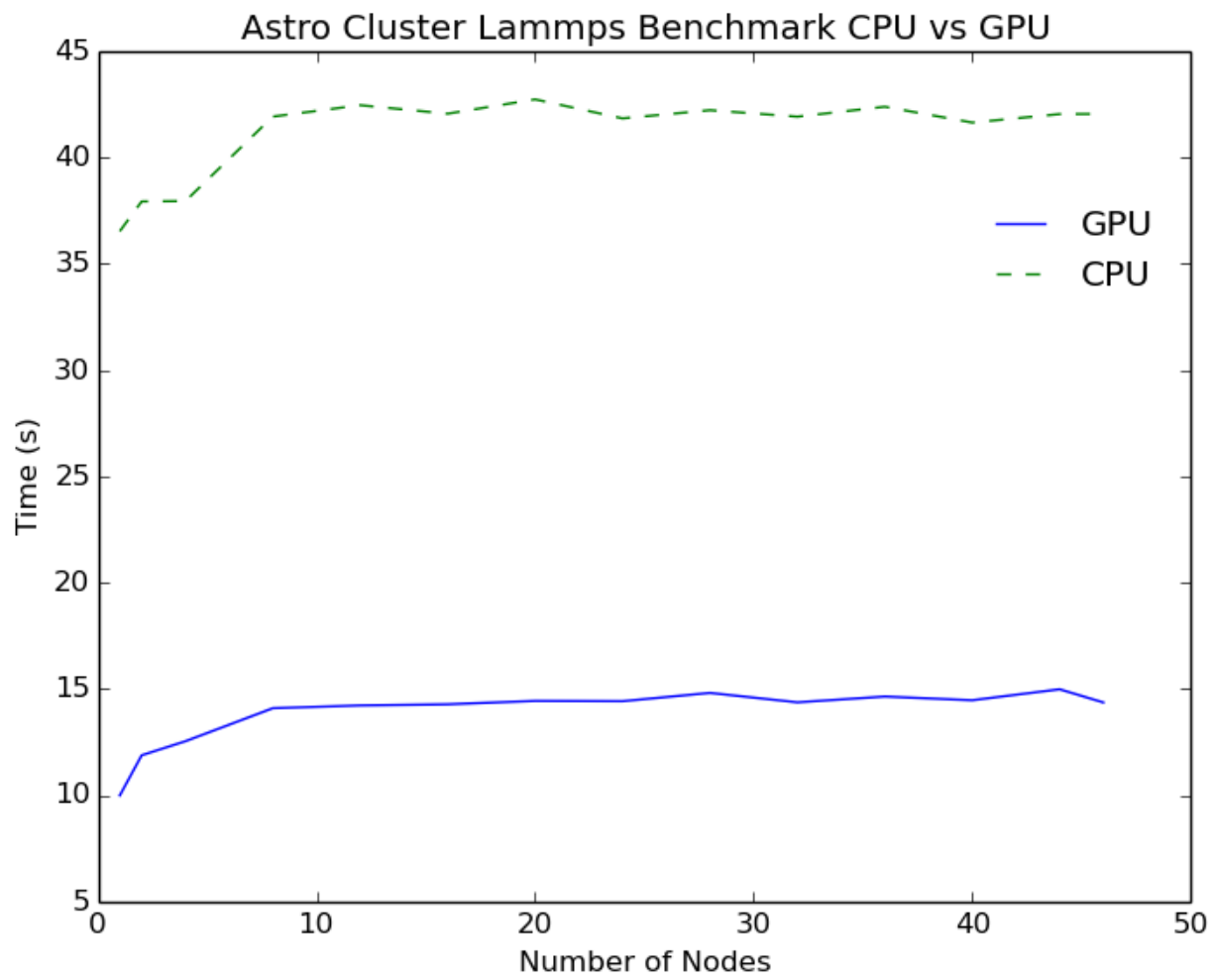

Figure 4.8: LAMMPS Benchmark GPU vs CPU runtimes 


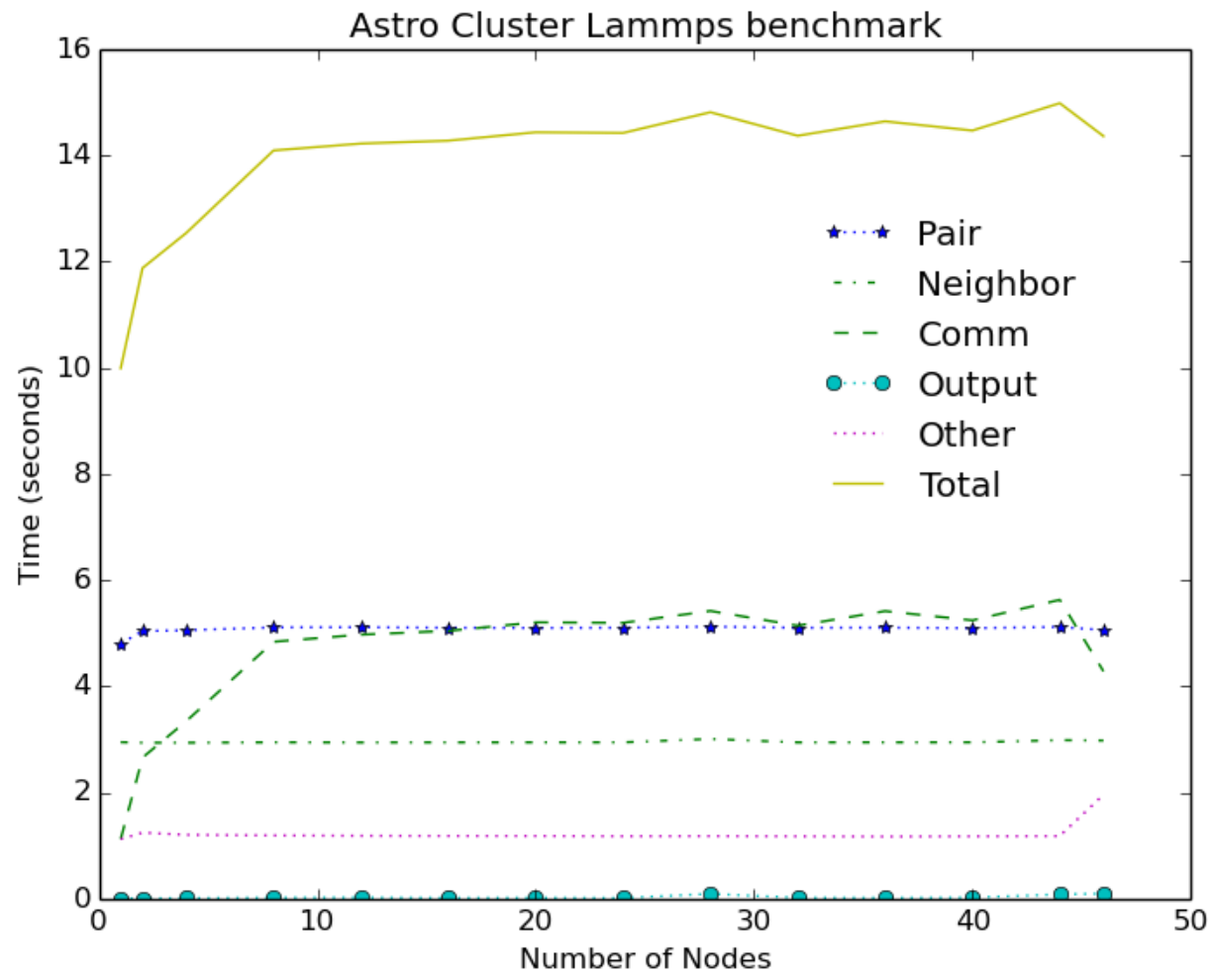

Figure 4.9: LAMMPS GPU benchmark with Communication Time 


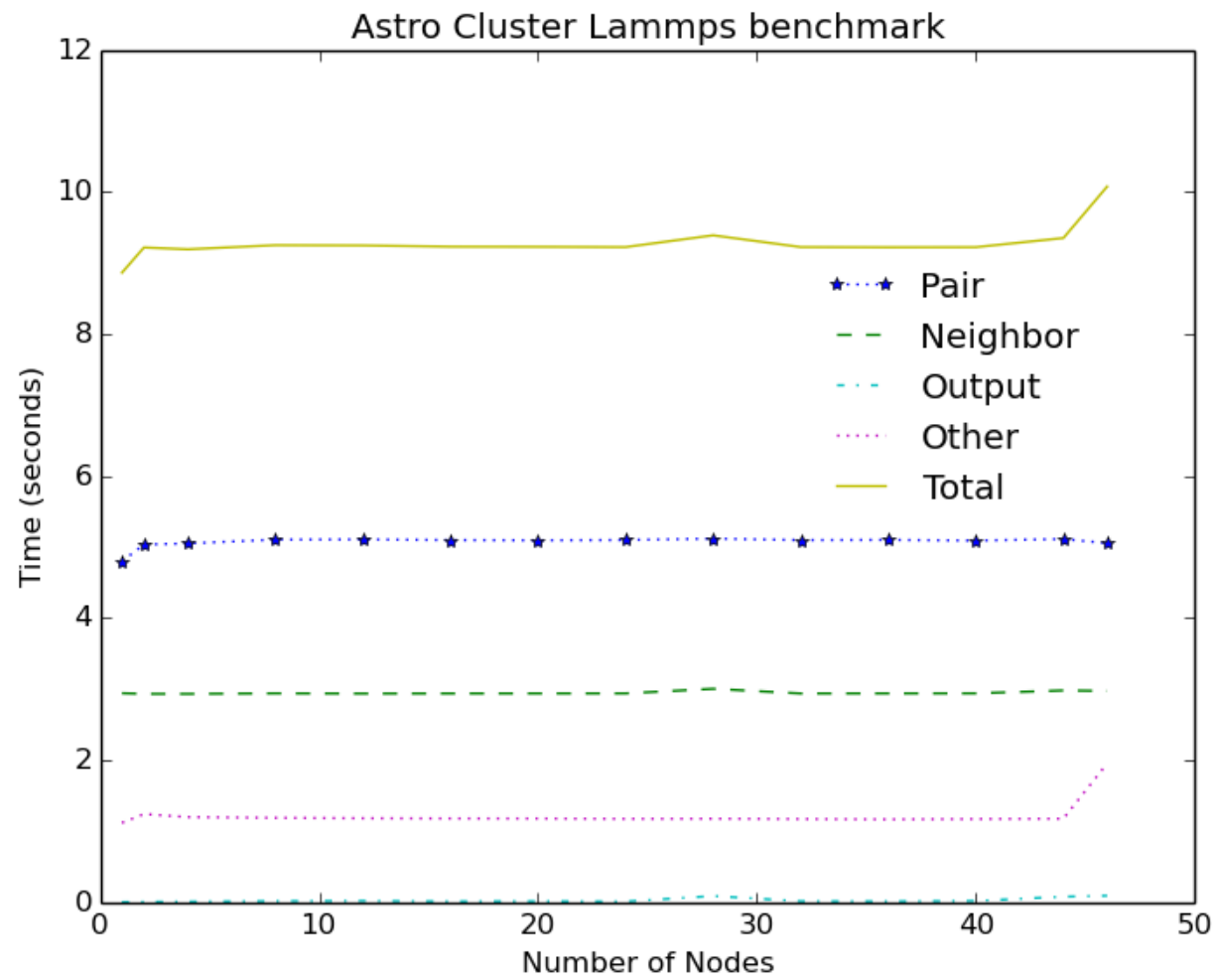

Figure 4.10: Run time with no Communication Times 


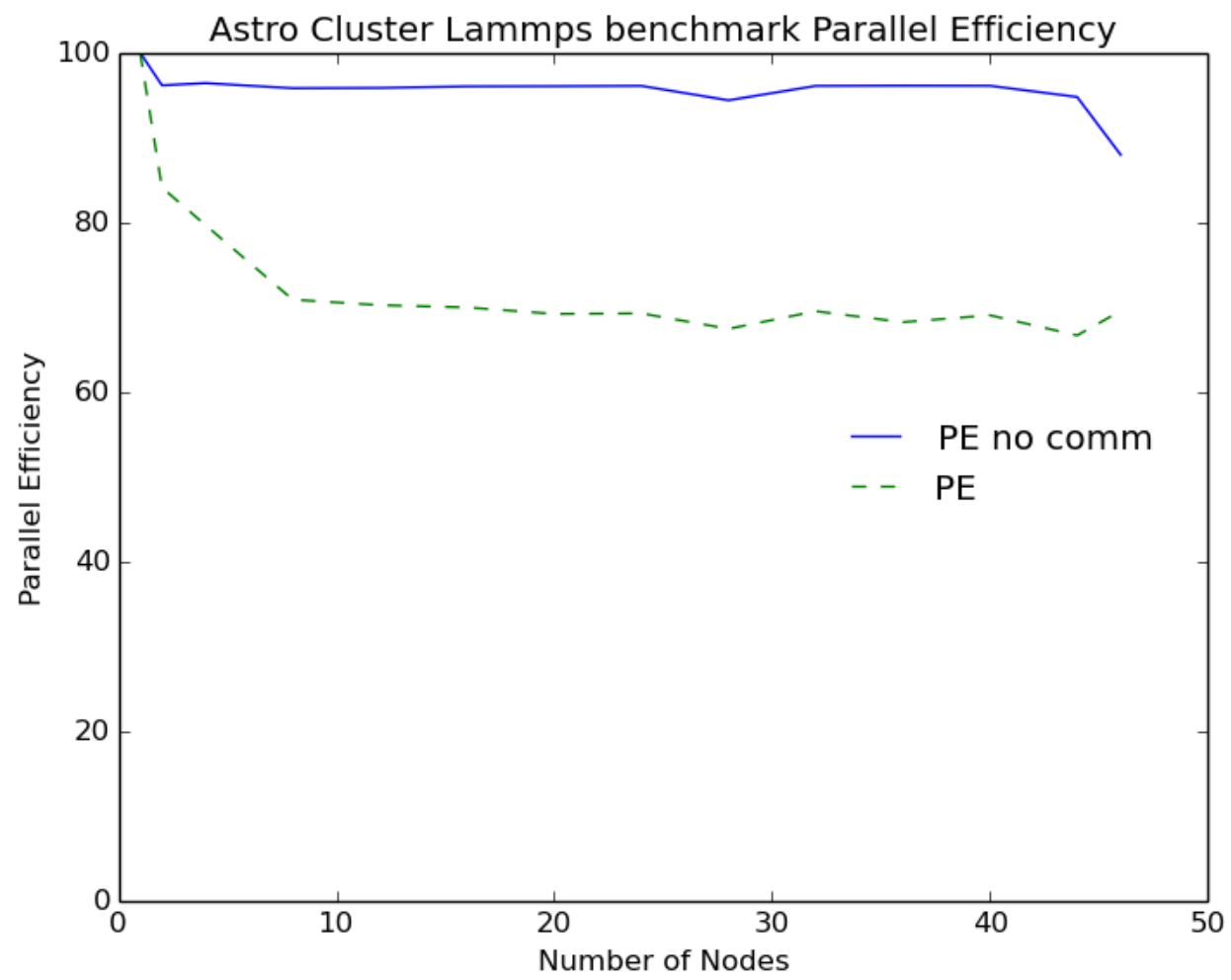

Figure 4.11: Parallel Efficiency of the LAMMPS benchmark 
culated. Parallel efficiency refers to how well the cluster scales as we increase the number of nodes in the cluster. This means that as the problem size and number of nodes scale up, the expected run-time should be the same in a perfectly efficient system.

The parallel efficiency of the cluster is shown in Figure 4.11. As we increase the number of nodes, the parallel efficiency drops slightly but still maintains strong efficiency and performs similar to other clusters as seen in [4].

Overall the system performs very well and and manages to maintain around $70 \%$ parallel efficiency at 46 nodes. With better network equipment, the cluster should be able to reach around $90 \%$ parallel efficiency at 46 nodes as shown by the tests with communication times removed. The parallel efficiency also drops slowly after its initial dip and proves that the cluster is scalable and can maintain high parallel efficiency even at larger node counts. 
Chapter 5

CONCLUSION

The Jetson TK1 shows great promise for use in a variety of compute intensive applications and distributed environments due to its low cost, low power consumption and hybrid CPU-GPU architecture. The Astro cluster demonstrates the viability of using the TK1 in a cluster setting and provides a proof-of-concept for future clusters planning on using low power CPU-GPU hybrids.

The Astro cluster is capable of running many of the same file systems used by top supercomputers, can be easily monitored using tools such as Ganglia, and can run many of the same HPC applications. The cluster uses only 810 Watts of power at peak performance and is capable of producing a peak performance of 326 GFLOPS of double-precision and 6800 GFLOPs of single-precision computing power, while maintaining $70 \%$ parallel efficiency. We also show that a simple cluster can be easily constructed and maintained for a relatively low cost.

The Astro cluster is also unique in that it is the largest TK1 cluster, and one of the few ARM-based clusters currently available. Research into low-power clusters is a relatively new topic, and Astro aims to provide an insight into how well these types of clusters might perform in the future. With the rise of faster and better low-power CPUs, many future clusters will likely utilize these types of architectures resulting in cheaper, more efficient clusters as shown by the Astro Cluster. 


\section{Chapter 6}

\section{FUTURE WORK}

The Astro cluster is a proof-of-concept for the viability of low-power ARM clusters and can be improved in numerous ways. The greatest improvement for the cluster would be better network equipment, which will allow us to increase the size of the cluster and provide better scaling statistics. The trade-off for this improvement is non-trivial additional cost.

Many of the benchmarks used in the system were also initially incompatible with the Jetson TK1 either due to its 32-bit limitation, or lack of CUDA support. For this paper, many of these benchmarks were modified to be able to run on the Jetson TK1, but not completely re-optimized. The benchmarks could likely perform significantly better if properly ported and tuned. We would also like to test a singleprecision version of the HPL benchmark to get proper statistics for single-precision performance.

While testing the Astro cluster, we also had some issues with reliability of the Jetson TK1. During long MPI tasks, or computation intensive applications, the nodes sometimes crashed and required a physical reboot to recover the node. Conducting a reliability test would be important to determine stability statistics of the Astro cluster. 


\section{BIBLIOGRAPHY}

[1] CephFS installation guide. Available at http://docs. ceph.com/docs/master/start/.

[2] GPM8212 power meter. Available at http://www.gwinstek.com/englobal/products/Other \_Meters/AC \_Power \_Meters/GPM-8212.

[3] iperf3: A TCP, UDP, and SCTP network bandwidth measurement tool. Available at https://github.com/esnet/iperf.

[4] LAMMPS. Available at http://lammps.sandia.gov/bench.html.

[5] MPICH. Available at http://www.mpich.org/.

[6] openBLAS library. Available at http://www. openblas.net/.

[7] OpenMPI. Available at https://www.open-mpi.org/.

[8] OrangeFS installation guide. Available at http://docs. orangefs. com/v_2_9/index.htm.

[9] Stress. Available at http://people.seas.harvard.edu/ apw/stress/.

[10] WiFi-220 temperature monitoring device. Available at http://www.temperaturealert.com/Temperature-Support/LegacyProduct-Support/WiFi-220. aspx.

[11] D. G. Andersen, J. Franklin, M. Kaminsky, A. Phanishayee, L. Tan, and V. Vasudevan. FAWN: A Fast Array of Wimpy Nodes. Commun. ACM, 54(7):101-109, July 2011. 
[12] B. Armstrong, H. Bae, R. Eigenmann, F. Saied, M. Sayeed, and Y. Zheng. HPC benchmarking and performance evaluation with realistic applications. In SPEC benchmarking workshop. Citeseer, 2006.

[13] R. Coker. Bonnie++ benchmark. Available at http: //www. coker. com.au/bonnie++/.

[14] S. J. Cox, J. T. Cox, R. P. Boardman, S. J. Johnston, M. Scott, and N. S. O’Brien. Iridis-pi: a low-cost, compact demonstration cluster. Cluster Computing, 17(2):349-358, 2013.

[15] J. J. Dongarra, P. Luszczek, and A. Petitet. The LINPACK benchmark: past, present and future. Concurrency and Computation: Practice and Experience, 15(9):803-820, 2003.

[16] E. M. Elnozahy, M. Kistler, and R. Rajamony. Energy-efficient server clusters. In Power-Aware Computer Systems, pages 179-197. Springer, 2002.

[17] J. L. Henning. Spec cpu2006 benchmark descriptions. ACM SIGARCH Computer Architecture News, 34(4):1-17, 2006.

[18] M. A. Heroux, D. W. Doerfler, P. S. Crozier, J. M. Willenbring, H. C. Edwards, A. Williams, M. Rajan, E. R. Keiter, H. K. Thornquist, and R. W. Numrich. Improving Performance via Mini-applications. Technical Report SAND2009-5574, Sandia National Laboratories, 2009.

[19] J. G. Koomey et al. Estimating total power consumption by servers in the US and the world, 2007.

[20] P. Koutoupis. The Lustre distributed filesystem. Linux J., 2011(210), Oct. 2011. 
[21] M. L. Massie, B. N. Chun, and D. E. Culler. The Ganglia distributed monitoring system: design, implementation, and experience. Parallel Computing, 30(7):817-840, 2004.

[22] J. D. McCalpin. STREAM benchmark. Available at https://www.cs.virginia.edu/stream/.

[23] D. Meisner, B. T. Gold, and T. F. Wenisch. PowerNap: eliminating server idle power. In ACM Sigplan Notices, volume 44, pages 205-216. ACM, 2009.

[24] P. M. Papadopoulos, M. J. Katz, and G. Bruno. NPACI: rocks: tools and techniques for easily deploying manageable Linux clusters. In Cluster Computing, 2001. Proceedings. 2001 IEEE International Conference on, pages 258-267, Oct 2001.

[25] F. Petrini, D. J. Kerbyson, and S. Pakin. The case of the missing supercomputer performance: Achieving optimal performance on the 8,192 processors of ASCI Q. In Supercomputing, 2003 ACM/IEEE Conference, pages 55-55. IEEE, 2003.

[26] Amazon Inc. Amazon web services. Available at https://aws .amazon.com/.

[27] ATLAS Project. Automatically tuned linear algebra software (atlas). Available at http://math-atlas. sourceforge.net/.

[28] Green500 Committee. Green500 list. Available at http://www.green500.org/.

[29] Intel Corporation. Intel mkl library. Available at https://software.intel.com/en-us/intel-mkl.

[30] NVIDIA Corporation. cuBLAS documentation. Available at https://developer.nvidia.com/cublas. 
[31] NVIDIA Corporation. Linux for Tegra R23.2 documentation. Available at https://developer.nvidia.com/embedded/linux-tegra.

[32] NVIDIA Corporation. NVIDIA CUDA Documentation. Available at http: //docs.nvidia.com/cuda/cuda-c-programming-guide.

[33] OpenMP Architecture Review Board. Openmp. Available at http: //openmp.org/.

[34] S. Rivoire, P. Ranganathan, and C. Kozyrakis. A comparison of high-level full-system power models. HotPower, 8:3-3, 2008.

[35] R. B. Ross, R. Thakur, et al. PVFS: A parallel file system for Linux clusters. In Proceedings of the 4th annual Linux showcase and conference, pages 391-430, 2000.

[36] R. B. Ross, R. Thakur, et al. PVFS: A parallel file system for Linux clusters. In Proceedings of the 4th annual Linux showcase and conference, pages 391-430, 2000.

[37] M. Rud and A. Pantiykchin. Development of GPU-accelerated localization system for autonomous mobile robot. In Mechanical Engineering, Automation and Control Systems (MEACS), 2014 International Conference on, pages 1-4, Oct 2014 .

[38] J. Shalf, S. Dosanjh, and J. Morrison. Exascale computing technology challenges. In High Performance Computing for Computational Science-VECPAR 2010, pages 1-25. Springer, 2010.

[39] A. L. Shimpi. Inside the Titan Supercomputer: 299K AMD x86 cores and 18.6K NVIDIA GPUs. AnandTech online computer hardware magazine, October, 2012. 
[40] M. Su, J. Tan, C.-Y. Lin, J. Ye, C.-H. Wang, and C.-L. Hung. Constructing a mobility and acceleration computing platform with NVIDIA Jetson TK1. In High Performance Computing and Communications (HPCC), 2015 IEEE 7th International Symposium on Cyberspace Safety and Security (CSS), 2015 IEEE 12th International Conferen on Embedded Software and Systems (ICESS), 2015 IEEE 17th International Conference on, pages 1854-1858, Aug 2015.

[41] Y. Ukidave, D. Kaeli, U. Gupta, and K. Keville. Performance of the NVIDIA Jetson TK1 in HPC. In Cluster Computing (CLUSTER), 2015 IEEE International Conference on, pages 533-534, Sept 2015.

[42] A. Vega, C.-C. Lin, K. Swaminathan, A. Buyuktosunoglu, S. Pankanti, and P. Bose. Resilient, UAV-embedded real-time computing. In Computer Design (ICCD), 2015 33rd IEEE International Conference on, pages 736-739, Oct 2015.

[43] S. A. Weil, S. A. Brandt, E. L. Miller, D. D. Long, and C. Maltzahn. Ceph: A scalable, high-performance distributed file system. In Proceedings of the 7th symposium on Operating systems design and implementation, pages 307-320. USENIX Association, 2006. 


\section{APPENDICES}

Appendix A

\section{DHCP CONFIGURATION}

DHCP configuration file for the Jetson Cluster. All nodes are assigned a static IP mapped ot their MAC address starting at 10.0.0.1. If more nodes are added, their MAC addresses need to be manually added to the list to keep IP assignment consistent.

The complete configuration file can be found at https://github.com/sksheen/ AstroScripts.

\# DHCPD Configuration for Jetson Cluster

\# All IPs are statically assigned by MAC address

ddns-update-style none;

\# DNS servers

option domain-name "Google";

option domain-name-servers 8.8.8.8;

\# Gateway, Desktop Computer that has internet access option routers 10.0.0.253;

default-lease-time -1 ;

max-lease-time -1 ;

subnet 10.0.0.0 netmask 255.255.255.0 \{ 
range $10.0 .0 .1 \quad 10.0 .0 .255 ;$

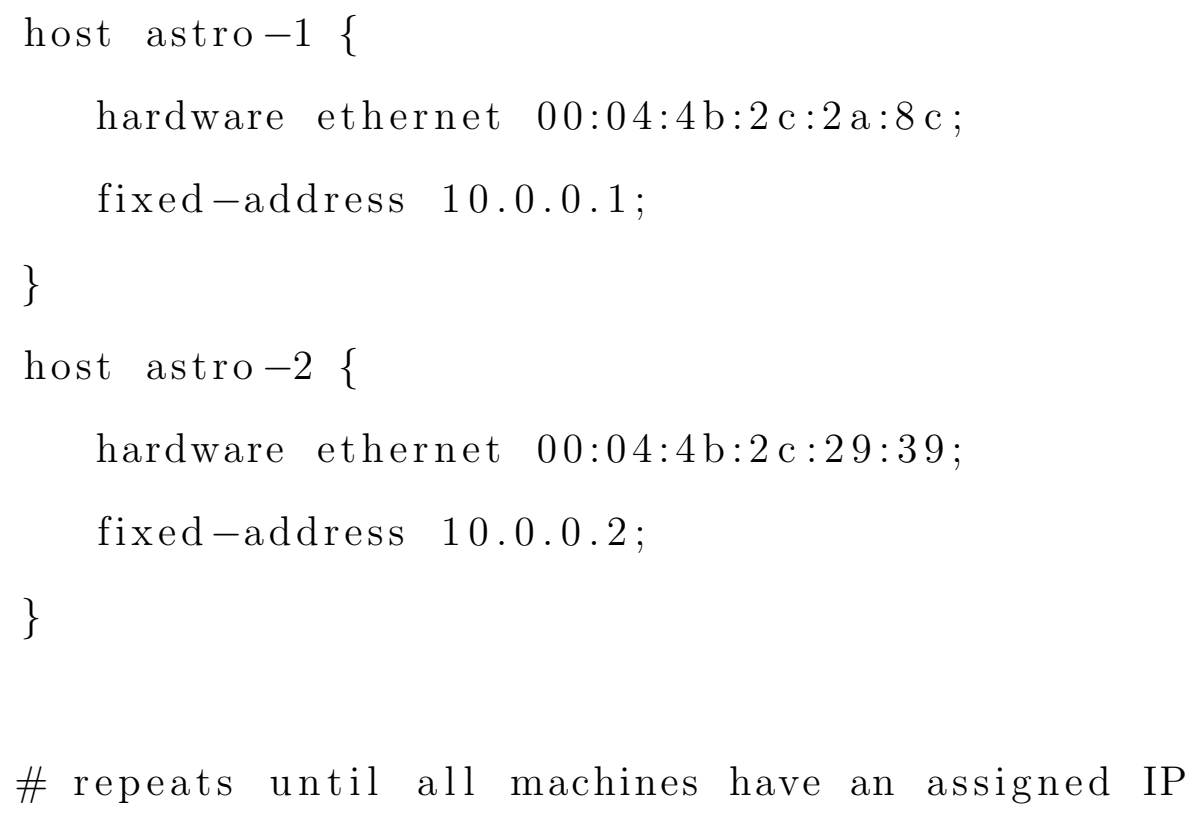




\section{Appendix B}

\section{GANGLIA CONFIGURATION}

Configuration file for gmond.conf. This sets up all ganglia clients to send data to the head node at IP address 10.0.0.253. If the node is at a different IP the configuration file needs to be modified.

The complete configuration file can be found at https://github.com/sksheen/ AstroScripts.

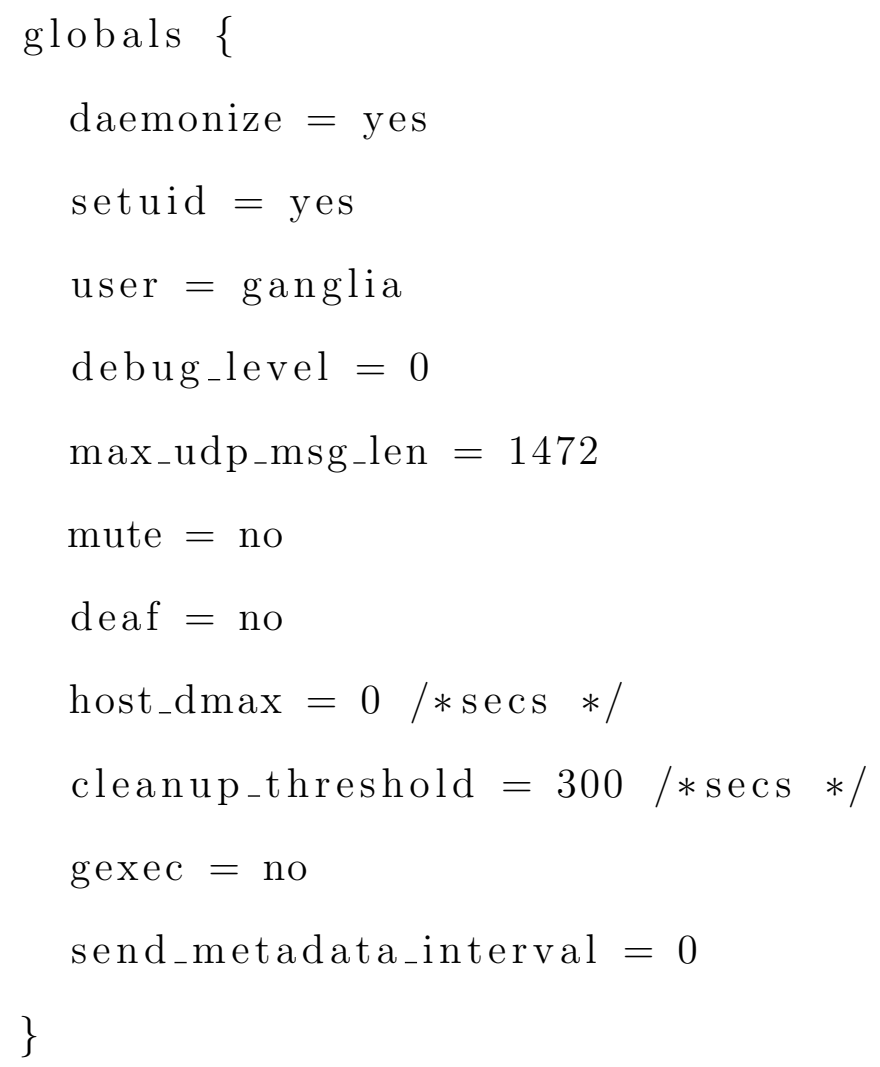




\section{Appendix C}

\section{CLUSTER SCRIPTS}

A collection of scripts used to manage libraries and detect node failures in the Astro Cluster is available at https://github.com/sksheen/AstroScripts. Scripts are commented and provide a variety of functionality for controlling the Cluster including creating a list of active IP addresses, sending bash scripts to all nodes, and shutting down or rebooting the cluster. 